\title{
Estimation of DSGE models when the data are persistent ${ }^{\text {is }}$
}

\author{
Yuriy Gorodnichenko ${ }^{\mathrm{a}, *}$, Serena $\mathrm{Ng}^{\mathrm{b}}$ \\ a Department of Economics, University of California, Berkeley, NBER, and IZA, USA \\ ${ }^{\mathrm{b}}$ Department of Economics, Columbia University, USA
}

\section{A R T I C L E I N F O}

\section{Article history:}

Received 9 October 2007

Received in revised form

17 February 2010

Accepted 19 February 2010

Available online 6 March 2010

\section{JEL classification:}

C32

$\mathrm{C} 5$

E32

\section{Keywords:}

Persistent data

Filters

Trends

Unit root

Spurious estimates

Business cycles

\begin{abstract}
A B S T R A C T
Dynamic stochastic general equilibrium (DSGE) models are often solved and estimated under specific assumptions as to whether the exogenous variables are difference or trend stationary. However, even mild departures of the data generating process from these assumptions can severely bias the estimates of the model parameters. This paper proposes new estimators that do not require researchers to take a stand on whether shocks have permanent or transitory effects. These procedures have two key features. First, the same filter is applied to both the data and the model variables. Second, the filtered variables are stationary when evaluated at the true parameter vector. The estimators are approximately normally distributed not only when the shocks are mildly persistent, but also when they have near or exact unit roots. Simulations show that these robust estimators perform well especially when the shocks are highly persistent yet stationary. In such cases, linear detrending and first differencing are shown to yield biased or imprecise estimates.
\end{abstract}

(c) 2010 Elsevier B.V. All rights reserved.

\section{Introduction}

Dynamic stochastic general equilibrium (DSGE) models are now accepted as the primary framework for macroeconomic analysis. Until recently, counterfactual experiments were conducted by assigning the parameters of the models with values that are loosely calibrated to the data. More recently, serious efforts have been made to estimate the model parameters using classical and Bayesian methods. This permits researchers to assess how well the models fit the data both in and out of samples. Formal estimation also permits errors arising from sampling or model uncertainty to be explicitly accounted for in counterfactual policy simulations. Arguably, DSGE models are now taken more seriously as a tool for policy analysis because of such serious econometric investigations.

Any attempt to estimate DSGE models must confront the fact that macroeconomic data are highly persistent. This fact often requires researchers to take a stand on the specification of the trends in DSGE models. Specifically, to take the model to the data, a researcher needs to use sample analogs of the deviations from steady states and, in doing so, must decide how to detrend the variables in the model and in the data. Table 1 is a non-exhaustive listing of how trends are treated in

\footnotetext{
This paper was presented at Brown University, the University of Michigan, the 2007 NBER Summer Institute, Princeton University, UC Berkeley, and the New York Area Macro Conference. We thank Marc Giannone, Tim Cogley, Anna Mikusheva, and two anonymous referees, the Associate Editor and the Editor for many helpful comments. The second author acknowledges financial support from the National Science Foundation (SES 0549978).

* Corresponding author.

E-mail address: ygorodni@econ.berkeley.edu (Y. Gorodnichenko).
} 
Table 1

Summary of selected work.

\begin{tabular}{|c|c|c|c|c|c|}
\hline Paper & Equations & Forcing variable & Model filter & Data filter & Estimator \\
\hline Kydland and Prescott (1982) & System & $\operatorname{ARMA}(1,1)$ & LT & $\mathrm{HP}$ & Calibration \\
\hline Altug (1989) & System & $\mathrm{I}(1)$ & $\mathrm{FD}_{1}$ & $\mathrm{FD}_{1}$ & MLE \\
\hline Christiano and Eichenbaum (1992) & System & $\mathrm{I}(1)$ & $z_{t}$ & HP & GMM \\
\hline Burnside et al. (1993) & System & $\mathrm{AR}(1)$ & LT & HP & GMM \\
\hline Burnside and Eichenbaum (1996) & System & $\mathrm{I}(1)$ & $z_{t}$ & $\mathrm{HP}$ & GMM \\
\hline McGrattan et al. (1997) & System & $\operatorname{VAR}(2)$ & LT & LT,HP & MLE \\
\hline Fuhrer (1997) & Equation & Not specified & Not specified & HP,LT,QT & GMM \\
\hline Clarida et al. (2000) & Equation & $\mathrm{AR}(1)$ & Not specified & LT,HP,CBO & GMM \\
\hline $\operatorname{Kim}(2000)$ & System & $\operatorname{AR}(1)$ & LT & LT & MLE \\
\hline Ireland (2001) & System & $\mathrm{AR}(1)$ & LT & LT & MLE \\
\hline Smets and Wouters (2003) & System & $\mathrm{AR}(1)$ & LT & $\mathrm{HP}$ & Bayesian \\
\hline $\operatorname{Dib}(2003)$ & System & $\operatorname{AR}(1)$ & LT & LT & MLE \\
\hline Fuhrer and Rudebusch (2004) & Equation & Not specified & Not specified & HP,CBO,QT & MLE,GMM \\
\hline Lubik and Schorfheide (2004) & System & $\operatorname{AR}(1)$ & LT & HP,LT & Bayesian \\
\hline Altig et al. (2004) & System & $\operatorname{ARI}(1,1)$ & $\mathrm{FD}_{1}$ & $\mathrm{FD}_{1}$ & GMM \\
\hline Ireland (2004) & System & $\mathrm{I}(1)$ & $\mathrm{FD}_{1}$ & $\mathrm{FD}_{1}$ & MLE \\
\hline Bouakez et al. (2005) & System & $\mathrm{AR}(1)$ & LT & LT & MLE \\
\hline Christiano et al. (2005) & System & Not specified & Not specified & VAR & GMM \\
\hline Del Negro et al. (2007) & System & $\operatorname{ARI}(1,1)$ & $\mathrm{FD}_{1}$ & $\mathrm{FD}_{1}$ & Bayesian \\
\hline Faia (2007) & System & $\mathrm{AR}(1)$ & LT & $\mathrm{HP}$ & Calibration \\
\hline Smets and Wouters (2007) & System & $\mathrm{AR}(1)$ & FD & FD & Bayesian \\
\hline
\end{tabular}

Note: CBO denotes actual series minus the Congress Budget Office's measure of potential output. I(1) and ARI(1,1) denote forcing variables with stochastic trends. AR and ARMA denote trend stationary forcing variables. VAR denotes filtering with a vector autoregression which can accommodate trend and difference stationary processes. FD is first differencing, $\mathrm{FD}_{1}$ is first differencing with the restriction that the forcing variable has a unit root (e.g., $\rho_{z}=1$ ), $\mathrm{LT}$ is projection on linear time trend, QT is projection on quadratic time trend, HP is Hodrick-Prescott filter, $z_{t}$ is detrending by the level of technology. The second column shows whether a paper estimates a system of equations ("system") or a single structural equation ("equation").

some notable papers. ${ }^{1}$ Some studies assume stochastic trends for the model and use first differenced data in estimation. A number of studies specify deterministic trends for the model and use linearly detrended data in estimation. Studies that apply the Hodrick-Prescott (HP) filter to the data differ in what trends are specified for the model. Some assume simple linear trends, while others assume unit root processes. Table 1 demonstrates that a variety of trends have been specified for the model and a variety of detrending methods have been used in estimation.

The problem for researchers is that it is not easy to ascertain whether highly persistent data are trend stationary or difference stationary in finite samples. While many have studied the implications for estimation and inference of inappropriate detrending in linear models, ${ }^{2}$ much less is known about the effects of detrending in estimation of non-linear models. From simulation evidence of Doorn (2006) for an inventory model, it seems that HP filtering can significantly bias the estimated dynamic parameters. While the local-to-unit framework is available to help researchers understand the properties of the estimated autoregressive root when the data are strongly persistent, it is unclear to what extent the framework can be used in non-linear estimation even in the single equation case. What makes estimation of DSGE models distinct is that they consist of a system of equations and misspecification in one equation can affect estimates in other equations.

This paper develops robust estimation procedures that do not require researchers to take a stand on whether shocks in the model have an exact or a near unit root, and yet obtain consistent estimates of the model parameters. All robust procedures have two characteristics. First, the same transformation (filter) is applied to both the data and the model variables. Second, the filtered variables are stationary when evaluated at the true parameter vector. The estimators have the classical properties of being $\sqrt{T}$ consistent and asymptotically normal for all values of the largest autoregressive root.

Our point of departure is that the rather common practice of applying different filters to the model variables and the data can have undesirable consequences. As will be shown later, estimates of parameters governing the propagation and amplification mechanisms in the model can be severely distorted when the trend specified for the model is not consistent with the one applied to the data. We insist on estimators that apply the same transformation to both the model and the data. This, however, may still lead to biased estimates if the filter does not remove the trends actually present in the data. Accordingly, one needs to work with filters that can remove both deterministic and stochastic trends without the researcher

\footnotetext{
${ }^{1}$ As of June 2009, these papers were cited almost 2500 times at the Web of Science (former Social Science Citation Index) and almost 8000 times at Google Scholar.

2 For example, Nelson and Kang (1981) showed that linear detrending a unit root process can generate spurious cycles. Cogley and Nason (1995a) found that improper filtering can alter the persistence and the volatility of the series while spurious correlations in the filtered data was documented in Harvey and Jaeger (1993). Singleton (1988) and Christiano and den Haan (1996) discussed how inappropriate filtering can affect estimation and inference in linear models.
} 
taking a stand before solving and estimating the model. The idea of applying robust filters to both the model and the data is not new. Christiano and den Haan (1996) as well as Burnside (1998) applied the HP filter to both the model and the data, but they had to resort to estimation by simulations to get around the large state vector that the HP filter induces. The filters to be considered have the same desirable feature as the HP in that they adapt to the trends in the data. However, they can be implemented with simple modifications to the state space system while keeping the dimension of the state vector small. Specifically, four transformations are considered: (i) quasi-differencing, (ii) unconstrained first differencing, (iii) hybrid differencing, and (iv) the HP filter. All filters can be used in GMM estimation but not every method can be implemented in the likelihood framework. Importantly, one can use standard asymptotic inference as the finite sample distribution of the estimators are well approximated by the normal distribution not only when the large autoregressive root is far from one, but also when it is near or on the unit circle. The procedures can be applied to DSGE models whose solution can be shown to exist and is unique, and can be solved using variations of the method discussed in Blanchard and Kahn (1980) and Sims (2002).

As discussed in Iskrev (2010) and Komunjer and $\mathrm{Ng}$ (2009), DSGE models are susceptible to identification failure, in which case, consistent estimation of parameters is not possible irrespective of the treatment of trends. In view of this consideration and to fix ideas, this paper uses a simple stochastic growth model whose properties are well understood. The model, which will be presented in Section 2, will also be used to perform baseline simulation experiments. The new estimators are presented in Sections 3 and 4. Discussion of the related literature is in Section 4 . Sections 5 and 6 use simulations to show that the robust approaches perform well especially when the shocks are highly persistent yet stationary. These results also hold up in larger models though some filters are more sensitive to the number of shocks than others. In contrast, linear detrending and first differencing often lead to severely biased estimates. Implementation issues are discussed in Section 7. Section 8 concludes.

\section{Preliminaries}

Consider the one sector stochastic growth model. The problem facing the central planner is

$$
\max E_{t} \sum_{t=0}^{\infty} \beta^{t}\left(\log C_{t}-\theta L_{t}\right)
$$

subject to feasibility and technological constraints

$$
\begin{aligned}
& Y_{t}=C_{t}+I_{t}=K_{t-1}^{\alpha}\left(Z_{t} L_{t}\right)^{(1-\alpha)} \\
& K_{t}=(1-\delta) K_{t-1}+I_{t} \\
& Z_{t}=\exp (\bar{\gamma} t) \exp \left(u_{t}^{z}\right), \quad u_{t}^{z}=\rho_{z} u_{t-1}^{z}+e_{t}^{z}, \quad\left|\rho_{z}\right| \leq 1
\end{aligned}
$$

Let $\widehat{m}_{t}=\left(\widehat{c}_{t}, \widehat{k}_{t}, \widehat{l}_{t}\right)=\left(c_{t}-\bar{g} t, k_{t}-\bar{g} t, l_{t}\right)=m_{t}-m_{z}^{*}$ where $Y_{t}$ is output, $C_{t}$ is consumption, $K_{t}$ is capital, $L_{t}$ is labor input, $Z_{t}$ is the level of technology, $e_{t}^{z}$ is an innovation in technology. Note that $\rho_{z}$ is allowed to be on the unit circle. Let lower case letters denote the natural logarithm of the variables, e.g. $c_{t}=\log C_{t}$. Let $c_{t}^{*}$ be such that $c_{t}-c_{t}^{*}$ is stationary; $k_{t}^{*}$ and $z_{t}^{*}$ are similarly defined. By assumption, labor $L_{t}$ is stationary for all $\rho_{z} \leq 1$ and thus $l_{t}^{*}=0$. Collect the observed model variables into the vector $m_{t}=\left(c_{t}, k_{t}, l_{t}\right)$ and denote the trend component of the model variables by $m_{t}^{*}=\left(c_{t}^{*}, k_{t}^{*}, l_{t}^{*}\right)$. In general, how $m_{t}^{*}$ is defined, how the model is linearized and estimated will depend on whether $\rho_{z}<1$ or $\rho_{z}=1$. Solving the system of expectational equations yields the reduced form

$$
\begin{aligned}
& \widehat{m}_{t}=\Pi \widehat{m}_{t-1}+B u_{t}^{z} \\
& u_{t}^{z}=\rho_{z} u_{t-1}^{z}+e_{t}^{z}
\end{aligned}
$$

As all roots of $\Pi$ are assumed to be strictly less than one, non-stationarity can only arise because $\rho_{z}$ is on the unit circle. Note that when $\rho_{z}=1$, the model needs to be linearized and solved with $m_{t}^{*}=\left(u_{t}+\bar{g} t, u_{t}+\bar{g} t, 0\right)=\left(z_{t}, z_{t}, 0\right)$. Despite the fact that the permanent shock $u_{t}^{z}$ is now a part of $m_{t}^{*},(1)$ is still the reduced form representation for the levels of the linearly detrended variables. In other words, the reduced form representation for $\widehat{m}_{t}$ is continuous in $\rho_{z}$ even though how one arrives at this representation will depend on $\rho_{z}$. Hence, without loss of generality, the representation (1) will be always used in subsequent discussions for all values of $\rho_{z}$.

Note that by definition, $\widehat{m}_{t}$ is the linearly detrended component of the model variables $m_{t}$. In other words, $\widehat{m}_{t}$ is a model concept. Hereafter, let $d_{t}$ denote the data analog of $m_{t}$. For the stochastic growth model, $d_{t}=\left(c_{t}, k_{t}, l_{t}\right)$ are the data series. Let $\widehat{d}_{t}$ be obtained by removing deterministic trends from $d_{t}$. Then $\widehat{d}_{t}$ is the data analog of $\widehat{m}_{t}$.

\section{Robust estimators}

This section presents robust methods that do not require the researcher to take a stand on the properties of trends in the data. The stochastic growth model is used to illustrate the intuition behind the proposed methods. 
Many methods have been used to estimate DSGE models. ${ }^{3}$ Our focus will be a method of moments (MM) estimator that minimizes the distance between the second moments of data and the second moments implied by the model, as in Christiano and den Haan (1996) and Christiano and Eichenbaum (1992). Adaptation to likelihood based estimation will be discussed in Section 7.

Let $\Theta$ denote the unknown structural parameters of the model and partition $\Theta=\left(\Theta^{-}, \rho_{z}\right)$. The generical MM estimator can be summarized as follows. Step 1 applies a filter (if necessary) to $d_{t}$ and computes $\widehat{\Omega}^{d}(j)$, the estimated covariance matrix of the filtered series at lag $j$. Collect the data moments in the vector

$$
\widehat{\omega}^{d}=\left(\operatorname{vech}\left(\widehat{\Omega}^{d}(0)\right)^{\prime} \operatorname{vec}\left(\widehat{\Omega}^{d}(1)\right)^{\prime} \ldots \operatorname{vec}\left(\widehat{\Omega}^{d}(M)\right)^{\prime}\right)^{\prime}
$$

Step 2 solves the rational expectations model for a guess of $\Theta$. Compute $\Omega^{m}(j)$, the model implied autocovariances of the filtered $\widehat{m}_{t}$ analytically or by simulation. Collect the model moments in the vector

$$
\omega^{m}=\left(\operatorname{vech}\left(\Omega^{m}(0)\right)^{\prime} \operatorname{vec}\left(\Omega^{m}(1)\right)^{\prime} \ldots \operatorname{vec}\left(\Omega^{m}(M)\right)^{\prime}\right)^{\prime}
$$

Step 3 estimates the structural parameters as

$$
\widehat{\Theta}=\underset{\Theta}{\operatorname{argmin}}\left\|\widehat{\omega}^{d}-\omega^{m}(\Theta)\right\|
$$

The choice of moments in MM can be important for identification (see e.g. Canova and Sala, 2009). The unconditional autocovariances are used in this paper but matching impulse responses can also be considered. Although MM is somewhat less widely used than maximum likelihood estimators in the DSGE literature, it does not require parametric specification of the error processes and it is easy to implement. As will be discussed later, the more important reason for using MM is practical as it can be used with many popular filters.

The robust approaches considered here always apply the same filter to the model and the data so that the filtered variables are stationary when evaluated at the true parameter of $\rho_{z}$, which can be one or close to one. The statistical properties of $\widehat{\Theta}$ will depend on $\rho_{z}$ and the filters used. The next four subsections consider four filters. Section 4 then explores which of these have better finite samples properties. Properties of estimators that do not have these features will also be compared later.

\subsection{The $Q D$ estimator}

Let $\Delta^{\rho_{z}}=1-\rho_{z} L$ be the quasi-differencing (QD) operator and let $\Delta^{\rho_{z}} \widehat{m}_{t}=\left(1-\rho_{z} L\right) \widehat{m}_{t}$. Multiplying both sides of $(1)$ by $\Delta^{\rho_{z}}$ and using $u_{t}^{z}=\rho_{z} u_{t-1}^{z}+e_{t}^{z}$ gives

$$
\Delta^{\rho_{z}} \widehat{m}_{t}=\Pi \Delta^{\rho_{z}} \widehat{m}_{t-1}+B e_{t}^{z}
$$

Note that the error term in the quasi-differenced model is an i.i.d. innovation. As $\Delta^{\rho_{z}} \widehat{m}_{t}$ is stationary for all $\rho_{z} \leq 1$, its moments are well defined. In contrast, the moments of $\widehat{m}_{t}$ are not well defined when $\rho_{z}=1$. This motivates estimation of $\Theta$ as follows.

First, initialize $\rho_{z}$. Second, quasi-difference $\widehat{d}_{t}$ with $\rho_{z}$ to obtain $\Delta^{\rho_{z}} \widehat{d}_{t}$. Compute $\widehat{\Omega}_{\Delta^{\rho_{z}}}^{d}(j)=\operatorname{cov}\left(\Delta^{\rho_{z}} \widehat{d}_{t}, \Delta^{\rho_{z}} \widehat{d}_{t-j}\right)$, the sample autocovariance matrix of the quasi-differenced data at lag $j=0, \ldots, M$. Define $\widehat{Y}_{\Delta^{\rho_{z}}}^{d}(j)=\widehat{\Omega}_{\Delta^{\rho_{z}}}^{d}(j)-\widehat{\Omega}_{\Delta^{\rho_{z}}}^{d}(0)$ and let $\widehat{\omega}_{\Delta^{\rho_{z}}}^{d}=\left(\operatorname{vec}\left(\widehat{Y}_{\Delta^{\rho_{z}}}^{d}(1)\right)^{\prime}, \ldots, \operatorname{vec}\left(\widehat{Y}_{\Delta^{\rho_{z}}}^{d}(M)\right)^{\prime}\right)^{\prime}$. Third, for a given $\rho_{z}$ and $\Theta^{-}$, solve for the reduced form (1). Apply $\Delta^{\rho_{z}}$ to $\widehat{m}_{t}$ and compute $\Omega_{\Delta^{\rho_{z}}}^{m}(j), j=1, \ldots, M$, the model implied autocovariance matrices of the quasi-differenced variables. Let $Y_{\Delta^{\rho_{z}}}^{m}(j)=\Omega_{\Delta^{\rho_{z}}}^{m}(j)-\Omega_{\Delta^{\rho_{z}}}^{m}(0)$. Define $\omega_{\Delta^{\rho_{z}}}^{m}\left(\rho_{z}\right)=\left(\operatorname{vec}\left(Y_{\Delta^{\rho_{z}}}^{m}(1)\right)^{\prime}, \ldots, \operatorname{vec}\left(Y_{\Delta^{\rho_{z}}}^{m}(M)\right)^{\prime}\right)^{\prime}$. Fourth, find the structural parameters $\widehat{\Theta}_{Q D}=\operatorname{argmin}_{\Theta}\left\|\widehat{\omega}_{\Delta^{\rho_{z}}}^{d}\left(\rho_{z}\right)-\omega_{\Delta^{\rho_{z}}}^{m}(\Theta)\right\|$.

The QD estimator is based on the difference between the model and the sample autocovariances of the filtered variables, normalized by the respective variance matrix $\Omega_{\Delta^{\rho_{z}}}(0)$. The QD differs from a standard covariance estimator in one important respect. The parameter $\rho_{z}$ now affects both the moments of the model and the data since the latter are computed for the data quasi-differenced at $\rho_{z}$. As $\rho_{z}$ and $\Theta^{-}$are estimated simultaneously, the filter is data dependent rather than fixed. The crucial feature is that the quasi-transformed data are stationary when evaluated at the true $\rho_{z}$, which subsequently permits application of a central limit theorem. The normalization of the lagged autocovariances by the variance amounts to using the moments

$$
\operatorname{cov}\left(\Delta^{\rho_{z}} \widehat{d}_{t}, \Delta^{\rho_{z}} \widehat{d}_{t}-\Delta^{\rho_{z}} \widehat{d}_{t-j}\right)-\operatorname{cov}\left(\Delta^{\rho_{z}} \widehat{m}_{t}, \Delta^{\rho_{z}} \widehat{m}_{t}-\Delta^{\rho_{z}} \widehat{m}_{t-j}\right)
$$

for estimation. The $j$-th difference of $\Delta^{\rho_{z}} \widehat{d}_{t}$ is always stationary and ensures that the asymptotic distribution is well behaved. Finally, observe that since the model is solved in levels and the transformed variables are used only to compute moments, all equilibrium relationships between variables are preserved.

\footnotetext{
${ }^{3}$ This includes likelihood and Bayesian based methods (e.g. Fernandez-Villaverde and Rubio-Ramirez, 2007; Ireland, 1997), two-step minimum distance approach (e.g., Sbordone, 2006), as well as simulation estimation (e.g., Altig et al., 2004). Ruge-Murcia (2007) provides a review of these methods.
} 


\subsection{The FD estimator}

If $\Delta^{\rho_{z}} \widehat{m}_{t}$ is stationary when $\rho_{z} \leq 1$, the data vector is also stationary when quasi-differenced at $\rho_{z}=1$. Denote the firstdifferencing (FD) operator by $\Delta=1-L$ and consider the following estimation procedure. First, compute $\widehat{\Omega}_{\Delta}^{d}(j)=\operatorname{cov}\left(\Delta \widehat{d}_{t}, \Delta \widehat{d}_{t-j}\right)$, the sample autocovariance matrix of the first differenced data at lag $j=1, \ldots, M$. Define $\widehat{\omega}_{\Delta}^{d}=\left(\operatorname{vech}\left(\widehat{\Omega}_{\Delta}^{d}(1)\right)^{\prime}, \ldots, \operatorname{vec}\left(\widehat{\Omega}_{\Delta}^{d}(M)\right)^{\prime}\right)^{\prime}$. Second, for a given $\Theta$, solve for the reduced form $(1)$. Compute $\Omega_{\Delta}^{m}(j)$, the model implied autocovariance matrices of the first-differenced variables $\Delta \widehat{m}_{t}$. Define $\omega_{\Delta}^{m}=\left(\operatorname{vech}\left(\Omega_{\Delta}^{m}(0)\right)^{\prime}, \ldots, v e c\left(\Omega_{\Delta}^{m}(M)\right)^{\prime}\right)^{\prime}$. Third, find the structural parameters $\widehat{\Theta}_{F D}=\arg \min _{\Theta}\left\|\widehat{\omega}_{\Delta}^{d}-\omega_{\Delta}^{m}(\Theta)\right\|$.

To be clear, the autocovariances are computed for the first differenced data and the model variables, but $\rho_{z}$ is a free parameter which is estimated. Note that the QD and FD estimators are equivalent when $\rho_{z}=1$. The key difference between FD and QD is that FD is a fixed filter while the QD is a data dependent filter.

\subsection{The hybrid estimator}

One drawback of the FD estimator is that when $\rho_{z}$ is far from unity, over-differencing induces a non-invertible movingaverage component. The estimates obtained by matching a small number of lagged autocovariances may be inefficient. The QD estimator does not have this problem, but $\widehat{\Omega}_{\Delta^{\rho_{z}}}^{d}(j)$ is quadratic in $\rho_{z}$. As will be explained below, this is why $\widehat{\Omega}_{\Delta^{\rho_{z}}}^{d}(j)$ was normalized by $\widehat{\Omega}_{\Delta^{\rho_{z}}}^{d}(0)$. These considerations suggest a hybrid (HD) estimator.

First, transform the observed data to obtain $\Delta^{\rho_{z}} \widehat{d}_{t}$ (as in QD) and $\Delta \widehat{d}_{t}$ (as in FD). Second, compute $\widehat{\Omega}_{Q D, \Delta}^{d}(j)=\operatorname{cov}\left(\Delta^{\rho_{z}} \widehat{d}_{t}, \Delta \widehat{d}_{t-j}\right)$. Define $\widehat{\omega}_{Q D, \Delta}^{d}=\left(\operatorname{vec}\left(\widehat{\Omega}_{\mathrm{QD}, \Delta}^{d}(0)\right)^{\prime}, \ldots, \operatorname{vec}\left(\widehat{\Omega}_{\mathrm{Q}, \Delta}^{d}(M)\right)^{\prime}\right)^{\prime}$. Third, for a given $\Theta$, solve for the reduced form (1), and compute the model implied autocovariances between the quasi-differenced and the first differenced variables. Define $\omega_{Q D, 4}^{m}=\left(\operatorname{vec}\left(\Omega_{Q D, 4}^{m}(0)\right)^{\prime}, \ldots, \operatorname{vec}\left(\Omega_{Q D, 4}^{m}(M)\right)^{\prime}\right)^{\prime}$. Fourth, find the structural parameters $\widehat{\Theta}_{H D}=$ $\arg \min _{\Theta}\left\|\widehat{\omega}_{Q D, 4}^{d}\left(\rho_{z}\right)-\omega_{Q D, 4}^{m}(\Theta)\right\|$. Notice that $\widehat{\Omega}_{Q D, \Delta}^{d}(j)$ is now linear in $\rho_{z}$, unlike $\widehat{\Omega}_{\Delta^{\rho_{z}}}^{d}(j)$.

\subsection{The HP estimator}

Linear filters such as the HP and the bandpass can also remove deterministic and stochastic trends, see Baxter and King (1999) and King and Rebelo (1993). The HP detrended series is defined as

$$
H P(L) d_{t}=\frac{\lambda(1-L)^{2}\left(1-L^{-1}\right)^{2}}{1+\lambda(1-L)^{2}\left(1-L^{-1}\right)^{2}} d_{t}
$$

The estimator can be constructed as follows. First, compute the autocovariance matrices of the HP-filtered data $\widehat{\Omega}_{H P}^{d}(0), \ldots, \widehat{\Omega}_{H P}^{d}(M)$. Define $\widehat{\omega}_{H P}^{d}=\left(\operatorname{vech}\left(\widehat{\Omega}_{H P}^{d}(0)\right)^{\prime}, \ldots, v e c\left(\widehat{\Omega}_{H P}^{d}(M)\right)^{\prime}\right)^{\prime}$. Second, for a given guess of $\Theta$, solve for the reduced form (1), and compute $\Omega^{m}(j)$, the autocovariances of $\widehat{m}_{t}$. Apply the Fourier transform to obtain the spectrum for $\widehat{m}_{t}$ at frequencies $2 \pi s / T, s=0, \ldots, T-1$. Multiply the spectrum by the gain of the HP filter. Inverse Fourier transform to obtain $\Omega_{H P}(j)$, the autocovariances of the $\mathrm{HP}(\mathrm{L}) \widehat{m}_{t}$. Define $\omega_{H P}^{m}=\left(\operatorname{vech}\left(\Omega_{H P}^{m}(0)\right)^{\prime}, \ldots, v e c\left(\Omega_{H P}^{m}(M)\right)^{\prime}\right)^{\prime}$. Third, find the structural parameters $\widehat{\Theta}_{H P}=\arg \min _{\Theta}\left\|\widehat{\omega}_{H P}^{d}-\omega_{H P}^{m}(\Theta)\right\|$.

This approach is similar to Burnside (1998) who also first applies the HP filter to both the model and the data series, and then uses simulations to compute model-implied moments. Like the FD, $\rho_{z}$ does not enter the filter but both the filtered data and the filtered model variables are stationary for all $\rho_{z} \leq 1$. Note that HP filtering involves estimation of many more autocovariances than the other estimators considered above.

\section{Properties of the estimators}

Let $\widehat{\omega}_{j}^{d}$ generically denote the $j$-th sample moments of the filtered variables while $\omega_{j}^{m}(\Theta)$ denote the model moment based on the same filter. Define $\bar{g}_{j}(\Theta)=\widehat{\omega}_{j}^{d}-\omega_{j}^{m}(\Theta)$ and let $\bar{g}(\Theta)=\left(\bar{g}_{0}(\Theta), \bar{g}_{1}(\Theta), \ldots, \bar{g}_{M}(\Theta)\right)$. Then the MM estimator $\widehat{\Theta}=\min _{\Theta}\|\bar{g}(\Theta)\|$ is a non-linear GMM estimator using an identity weighting matrix. This sub-optimal weighting matrix is used because when there are fewer shocks than variables in the system, stochastic singularity will induce collinearity in the variables resulting in a matrix of covariances that would be singular. Even if there are as many shocks as endogenous variables, Abowd and Card (1989), Altonji and Segal (1996) and others find that an identity matrix performs better than the optimal weighting matrix in the context of estimating covariance structures. The optimal weighting matrix, which contains high order moments, tends to correlate with the moments and this correlation undermines the performance of the estimator.

Let $\bar{G}(\Theta)$ be the matrix of derivatives of $\bar{g}(\Theta)$ with respect to $\Theta$. In standard covariance structure estimation, the parameters enter the model moments $\omega^{m}(\Theta)$ but not the sample $\widehat{\omega}^{d}$, so that if $\widehat{\omega}^{d}$ are moments of stationary variables, 
then under regularity conditions such as stated in Newey and McFadden (1994), the conventional result that $\widehat{\Theta}$ is consistent obtains. Furthermore,

$$
\sqrt{T}\left(\widehat{\Theta}-\Theta_{0}\right) \stackrel{d}{\longrightarrow} A \cdot N(0, S)
$$

where $A=\left(G_{0}^{\prime} G_{0}\right)^{-1} G_{0}^{\prime}, \sqrt{T} \bar{g}\left(\Theta_{0}\right) \stackrel{d}{\longrightarrow} N(0, S)$, and $G_{0}$ is the probability limit of $\bar{G}(\Theta)$ evaluated at $\Theta=\Theta_{0}$. This distribution theory applies to the FD and the HP because these two filters do not depend on unknown parameters and the filtered variables are always stationary. For the HD estimator, $\widehat{\omega}^{d}$ depends on $\rho_{z}$ but its first derivative does not, so that a quadratic expansion of the objective function can still be used to derive the asymptotic distribution of the estimator. Although $\bar{G}(\Theta)$ for the HD has a random limit when $\rho_{z}=1, \widehat{\omega}^{d}$ is a vector of covariances of stationary variables when evaluated at the true value of $\rho_{z}$. Thus, the 'standardized' HD estimator (or the $t$ statistic) remains asymptotically normal.

To understand the properties of the QD estimator, an explanation for why the lagged autocovariances are normalized by the variance is necessary. Suppose $\tilde{g}_{j}(\Theta)=\widehat{\Omega}_{\Delta^{\rho_{z}}}^{d}(j)-\Omega_{\Delta^{\rho_{z}}}^{m}(j)$ was used instead of $\bar{g}_{j}(\Theta)=\widehat{\omega}_{\Delta^{\rho_{z}}}^{d}(j)-\omega_{\Delta^{\rho_{z}}}^{m}(j)$ where $\omega_{\Delta^{\rho_{z}}}^{d}(j)=\widehat{\Omega}_{\Delta^{\rho_{z}}}^{d}(j)-\widehat{\Omega}_{\Delta^{\rho_{z}}}^{d}(0)$, and $\omega_{\Delta^{\rho_{z}}}^{m}$ is likewise defined. Minimizing $\|\tilde{g}(\Theta)\|$ over $\Theta$ yields an estimator, say, QD ${ }^{0}$. The problem here is that $\widehat{\Omega}_{\Delta^{\rho_{z}}}^{d}(j)$ is a cross-product of data quasi-differenced at $\rho_{z}$, and is thus quadratic in $\rho_{z}$. The quadratic expansion of $\|\tilde{g}(\Theta)\|$ around $\Theta_{0}$ contains terms that are not negligible when $\rho_{z}$ is one. As such, the sample objective function cannot be shown to converge uniformly to the population objective function. Gorodnichenko et al. (2009) show in a simpler setting that the $\mathrm{QD}^{0}$ estimator for $\rho_{z}$ is consistent but it has a convergence rate of $T^{3 / 4}$ and is not asymptotically normal. The QD estimator is motivated by the fact that the offending term in the quadratic expansion of $\widehat{\Omega}_{\Delta^{\rho_{z}}}^{d}(j)$ is collinear with $\widehat{\Omega}_{\Delta^{\rho_{z}}}^{d}(0)$ when $\rho_{z}=1$.

Proposition 1. Consider a DSGE model whose reduced form is given by (1) and all roots of $\Pi$ less than one. Let $\Theta$ be the unknown parameters of the model and let $\widehat{\Theta}^{Q D}$ be the $Q D$ estimator of $\Theta$. Then $\sqrt{T}\left(\widehat{\Theta}^{Q D}-\Theta_{0}\right) \stackrel{d}{\longrightarrow} N\left(0, A v a r\left(\widehat{\Theta}^{Q D}\right)\right)$.

A sketch of the argument is given in the supplementary material for the baseline model whose closed-form solution is known. By subtracting the variance from each lagged autocovariance, the quadratic terms in the expansion of the objective function are asymptotically negligible. This leads to the rather unexpected property that $\widehat{\Theta}$ is asymptotically normal even when $\rho_{z}=1$. From a practical perspective, the primary advantage of the robust estimators is that when properly studentized, the estimators are normally distributed whether $\rho_{z}<1$ or $\rho_{z}=1$, which greatly facilitates inference. Since all estimators are consistent and asymptotically normal, it remains to consider which estimator is more efficient in finite samples.

\subsection{Related literature}

There is a small literature on estimation of non-linear dynamic models when the data are highly persistent. Cogley (2001) considers several estimators and finds that using cointegration relationships in the unconditional Euler equations works quite well. Our method is similar to Cogleys (2001) in that neither requires the researcher to take a stand on the properties of the trend function and yet the moments used in GMM estimation are always stationary. However, there are important differences. First, quasi-differencing can easily handle multiple I(1) or highly persistent shocks. In contrast, cointegration relationships can be used only for certain types of shocks. For example, if the shock to disutility of labor supply is an I(1) process, there is no cointegration vector to nullify a trend in hours. Second, cointegration often involves estimating identities and therefore the researcher has to add an error term (typically measurement error) to avoid singularity. We do not estimate specific equations and hence do not need to augment the model with additional, atheoretical shocks. Finally, some structural parameters such as adjustment costs cannot be identified by cointegration relations because they are zero by construction in the steady state. In contrast, the estimators proposed here utilize short-run dynamics in the data to estimate the parameters governing the short-run dynamics of the model.

Fukac and Pagan (2006) consider how the treatment of trends might affect estimation of DSGE models, but their analysis is confined to a single equation. They propose to use the Beveridge-Nelson decomposition to estimate and remove the permanent components in the data. This assumes that the restrictions implied by Beveridge-Nelson trend are consistent with the data. Canova (2008) explicitly treats the latent trends as unobserved components and estimates the trends and cycles directly. While this allows the data to select the trend endogenously, the procedure can be imprecise when the random walk component is small. Canova and Ferroni (2008) consider many filters and treat each as the true cyclical component measured with error. They are primarily concerned with the consequences of data filtering taking the model specification as given. This paper takes the view that the trends specified for the model should be consistent with the facts that we sought to explain. As such, it should not be taken as given. 
Table 2

Neoclassical growth model.

\begin{tabular}{|c|c|c|c|c|c|c|c|c|c|}
\hline$\rho_{z}$ & $\begin{array}{l}\text { Data filter } \\
\text { Model filer }\end{array}$ & $\begin{array}{l}\text { QD } \\
\text { QD } \\
(1)\end{array}$ & $\begin{array}{l}\text { HD } \\
\text { HD } \\
(2)\end{array}$ & $\begin{array}{l}\text { FD } \\
\text { FD } \\
(3)\end{array}$ & $\begin{array}{l}\text { HP } \\
\text { HP } \\
(4)\end{array}$ & $\begin{array}{l}\text { LT } \\
\text { LT } \\
(5)\end{array}$ & $\begin{array}{l}\mathrm{FD}_{1} \\
\mathrm{FD}_{1} \\
(6)\end{array}$ & $\begin{array}{l}\text { HP } \\
\text { LT } \\
(7)\end{array}$ & $\begin{array}{l}\mathrm{HP} \\
z_{t} \\
(8)\end{array}$ \\
\hline \multicolumn{10}{|c|}{ Estimate of $\alpha$} \\
\hline \multirow[t]{2}{*}{0.95} & Mean & 0.318 & 0.333 & 0.367 & 0.350 & 0.480 & 0.400 & 0.675 & 0.990 \\
\hline & St.dev. & 0.052 & 0.061 & 0.110 & 0.103 & 0.120 & 0.083 & 0.022 & \\
\hline \multirow[t]{2}{*}{0.99} & mean & 0.308 & 0.324 & 0.372 & 0.360 & 0.810 & 0.377 & 0.789 & 0.990 \\
\hline & St.dev. & 0.053 & 0.066 & 0.115 & 0.120 & 0.201 & 0.109 & 0.024 & \\
\hline \multirow[t]{2}{*}{1.00} & Mean & 0.304 & 0.312 & 0.349 & 0.351 & 0.905 & 0.357 & 0.817 & 0.990 \\
\hline & St.dev. & 0.054 & 0.061 & 0.105 & 0.115 & 0.183 & 0.113 & 0.022 & \\
\hline \multicolumn{10}{|c|}{ Estimate of $\rho_{z}$} \\
\hline \multirow[t]{2}{*}{0.95} & Mean & 0.949 & 0.949 & 0.950 & 0.950 & 0.914 & 1.000 & 0.541 & 1.000 \\
\hline & St.dev. & 0.006 & 0.014 & 0.017 & 0.015 & 0.042 & & 0.049 & \\
\hline \multirow[t]{2}{*}{0.99} & mean & 0.989 & 0.990 & 0.991 & 0.991 & 0.864 & 1.000 & 0.485 & 1.000 \\
\hline & St.dev. & 0.002 & 0.005 & 0.007 & 0.016 & 0.094 & & 0.041 & \\
\hline \multirow[t]{2}{*}{1.00} & Mean & 0.999 & 1.000 & 0.998 & 1.000 & 0.694 & 1.000 & 0.461 & 1.000 \\
\hline & St.dev. & 0.001 & 0.003 & 0.005 & 0.011 & 0.123 & & 0.039 & \\
\hline \multicolumn{10}{|c|}{ Estimate of $\sigma_{z}$} \\
\hline \multirow[t]{2}{*}{0.95} & Mean & 0.981 & 1.021 & 1.157 & 1.076 & 1.135 & 1.334 & 1.949 & 0.046 \\
\hline & St.dev. & 0.123 & 0.187 & 0.441 & 0.291 & 0.283 & 0.285 & 0.167 & 0.006 \\
\hline \multirow[t]{2}{*}{0.99} & Mean & 0.962 & 1.001 & 1.154 & 1.107 & 4.348 & 1.185 & 2.912 & 0.042 \\
\hline & St.dev. & 0.111 & 0.170 & 0.367 & 0.303 & 2.169 & 0.347 & 0.397 & 0.006 \\
\hline \multirow[t]{2}{*}{1.00} & Mean & 0.955 & 0.974 & 1.073 & 1.087 & 19.803 & 1.107 & 3.289 & 0.041 \\
\hline & St.dev. & 0.108 & 0.145 & 0.295 & 0.513 & 10.681 & 0.341 & 0.478 & 0.005 \\
\hline
\end{tabular}

Note: The number of simulations is 2000 . Sample size is $T=200$. LT is linear detrending, HP is Hodrick-Prescott filter, FD is first differencing, FD 1 is first differencing with the restriction that $\rho_{z}=1, \mathrm{QD}$ is quasi-differencing, HD is hybrid differencing, $z_{t}$ is detrending by the level of technology.

\section{Simulations: baseline model}

This section uses the stochastic growth model to conduct Monte Carlo experiments. Data are generated with either deterministic trends $\left(\rho_{z}<1\right)$ or stochastic trends $\left(\rho_{z}=1\right)$ using the model equations for $\widehat{m}_{t}$. The model variables are then rescaled back to level form and treated as observed data $d_{t}=\left(c_{t}, k_{t}, y_{t}, l_{t}\right)$, which are taken as given in estimation. The variance and the first order $(M=1)$ auto- and cross variance of the four variables are used as moments. Alternative choices of observed variables, such as excluding the capital stock series, yield very similar results.

The model is calibrated as follows: capital intensity $\alpha=0.33$; disutility of labor $\theta=1$; discount factor $\beta=0.99$; depreciation rate $\delta=0.1$; gross growth rate in technology $\bar{\gamma}=0.005$. There is only one shock in this baseline model. Thus, the standard deviation of $e_{t}^{z}$ is set to $\sigma_{z}=1$ without loss of generality. The admissible range of the estimates of $\alpha$ is $[0.01,0.99]$. The persistence parameter $\rho_{z}$ takes values $(0.95,0.99,1)$. To decouple the treatment of trends from the identification issues, the parameter vector $\Theta=(\alpha, \rho, \sigma)$ is estimated while $(\beta, \delta, \theta)$ is assumed known. ${ }^{4}$ In each of the 2000 replications for each parameter set, series with $T=200$ observations are created. Other sample sizes are also considered.

In all simulations and for all estimators, the starting values in optimization routines are equal to the true parameter values. The model is solved using the Anderson and Moore (1985) algorithm. Mildly explosive estimates are allowed because otherwise solutions for $\widehat{\rho}_{z}$ will be truncated to the right at one making the distribution of $\widehat{\rho}_{z}$ highly skewed. Only parameter values consistent with a unique rational expectations equilibrium are allowed. ${ }^{5}$

Table 2 reports simulation results for the baseline growth model. The persistence of technology shocks is given in the left column. The first and second rows indicate which filter is applied to both the data and the model variables. Columns (1)-(4) report results for the four estimators. By and large, all four filters yield estimates which are very close to the true values. Notice that while $\rho_{z}$ is always precisely estimated, the variance of the estimates varies substantially across filters. The QD estimates have the lowest standard deviation while the HP estimates are two to five times more variable than the QD. The HD is more precise than the FD but is less precise than the QD. This pattern is recurrent in all simulations.

Fig. 1 shows the root mean squared error (RMSE) for different estimators and sample sizes. The QD estimator performs the best while the FD tends to have the largest RMSE in almost all cases. In small samples, the HP tends to lead to large RMSE. However, in larger samples, the HP approaches the HD which is only slightly inferior to the QD.

\footnotetext{
${ }^{4}$ The average growth rate $\bar{g}$ is estimated in the preliminary when the series is projected on a linear time trend.

${ }^{5} \mathrm{~A}$ rational expectations solution is said to be stable if the number of unstable eigenvalues of the system equals the number of forward looking variables. Stability in this context refers to the internal dynamics of the system. This is distinct from covariance stationarity of the time series data, which obtains when $\rho_{z}<1$. It is possible for $\rho_{z}$ to be mildly explosive and yet the system has a stable, unique rational expectations equilibrium. Only a tiny fraction of simulations was discarded due to non-uniqueness of the rational expectations equilibrium.
} 

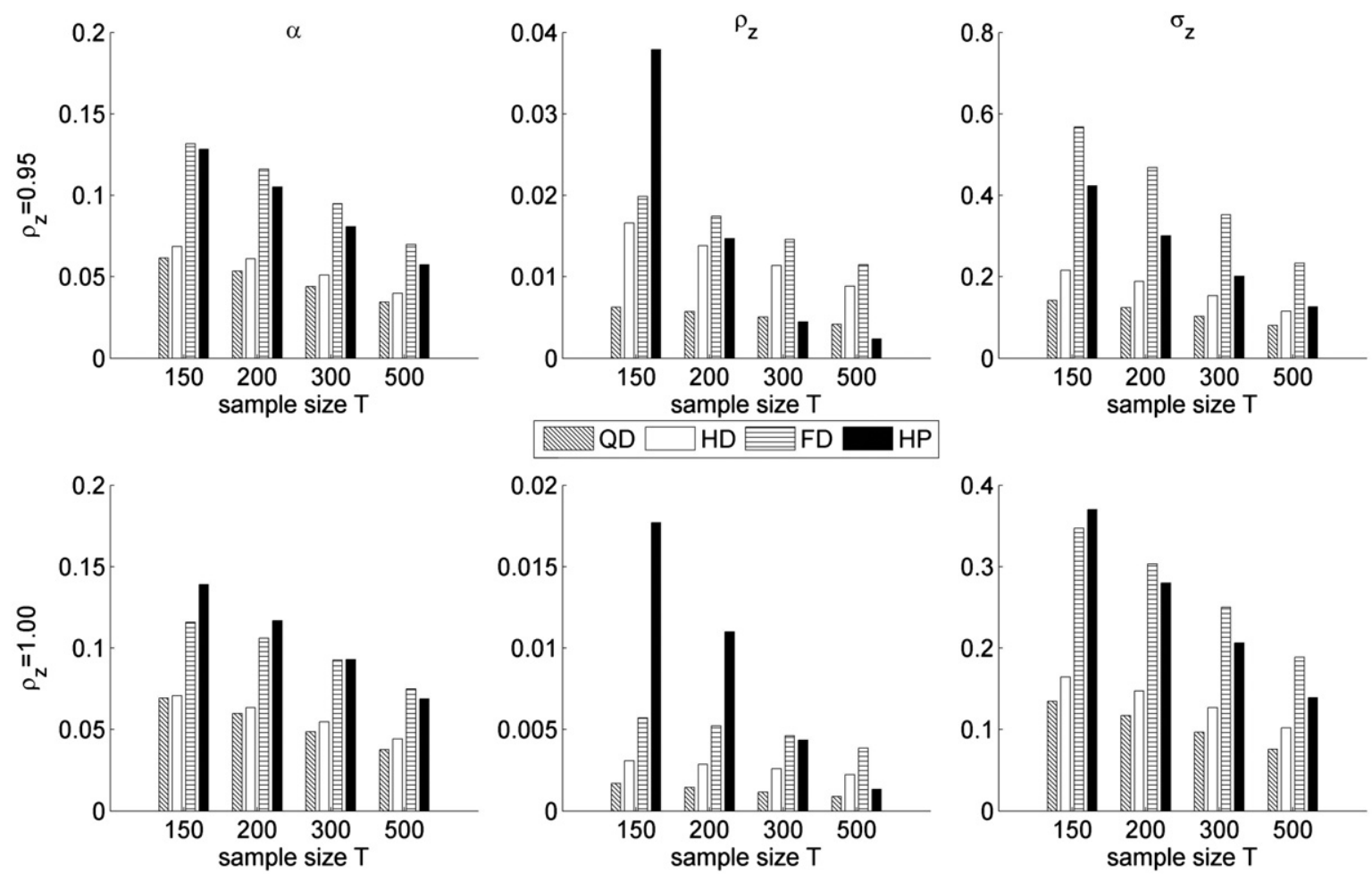

Fig. 1. Root mean squared errors. Note: This figure shows the root mean squared errors (RMSE) for four estimators which apply the same transformation (QD, HP, FD, HD) to data and model series. RMSE are shown for three parameter estimates: $\alpha$, elasticity of output with respect to capital; $\rho_{z}$, persistence of technology shocks; $\sigma_{z}$, standard deviation of technology shocks.
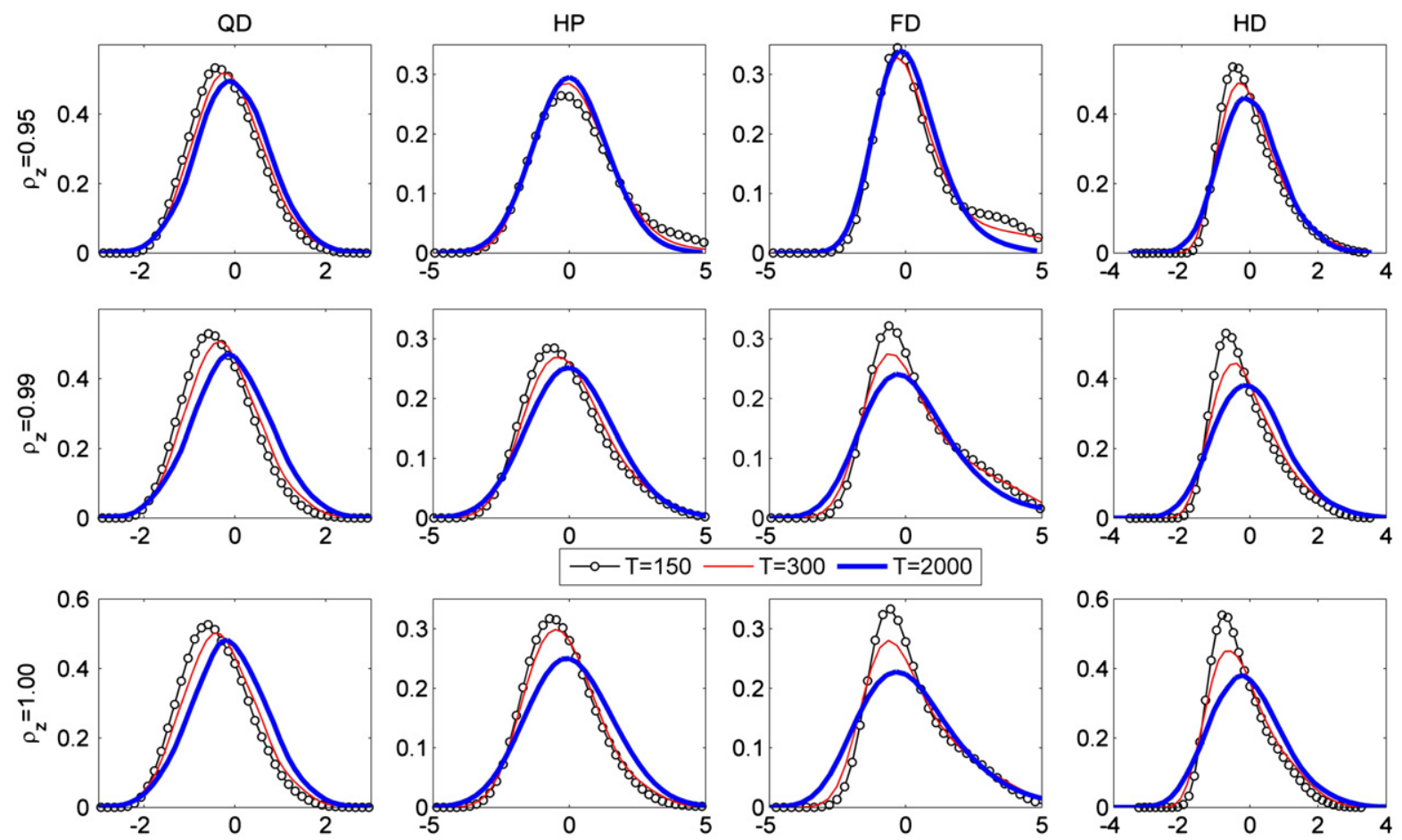

Fig. 2. Kernel density of simulated $\sqrt{T}(\widehat{\alpha}-\alpha)$. Note: This figure shows the kernel density of $\sqrt{T}(\widehat{\alpha}-\alpha)$ for four estimators which apply the same transformation (QD, HP, FD, HD) to data and model series. Kernel densities are shown for three sample sizes T=150, 300, and 2000. Parameter $\alpha$ is the elasticity of output with respect to capital. 

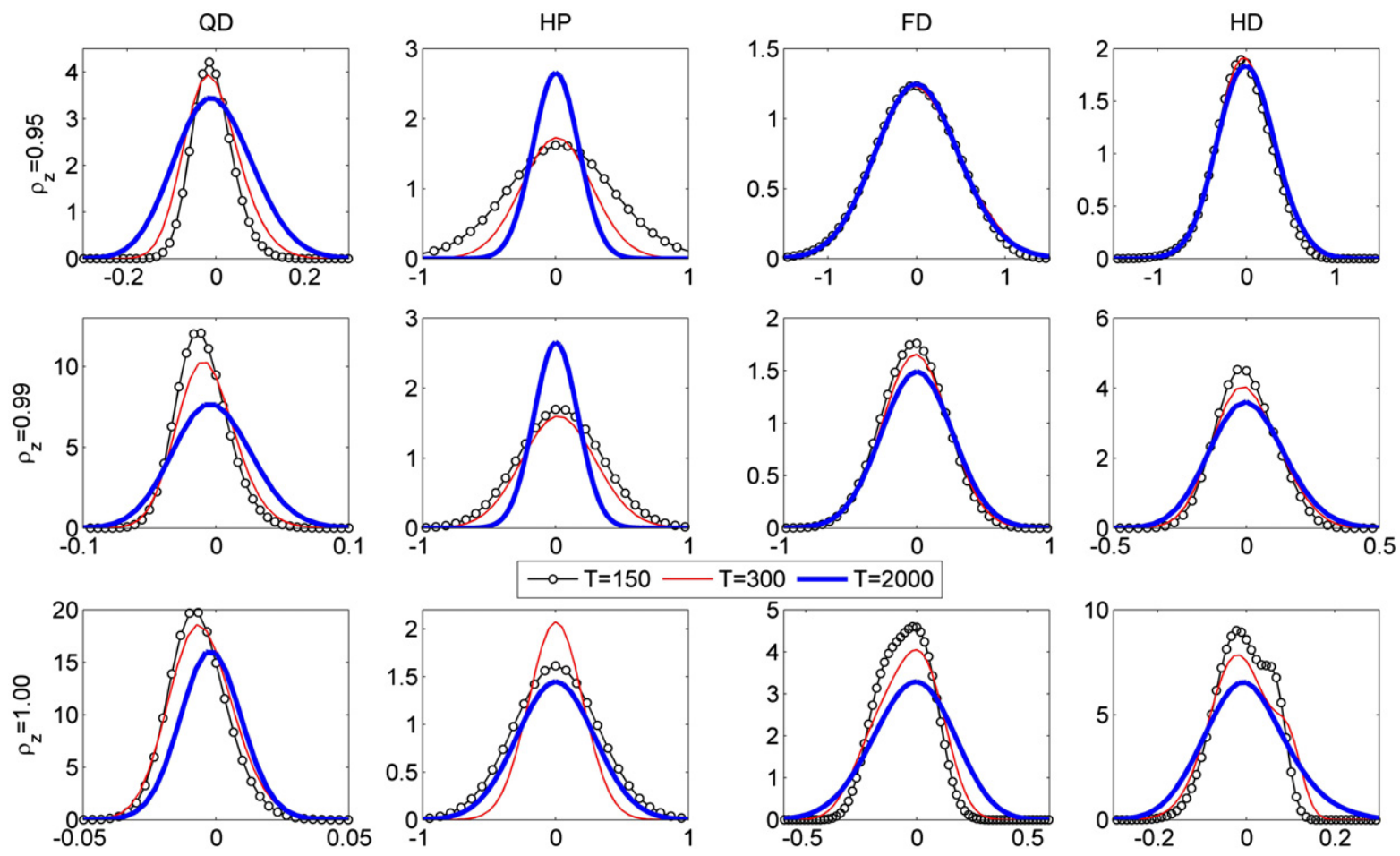

Fig. 3. Kernel density of simulated $\sqrt{T}\left(\widehat{\rho}_{z}-\rho_{z}\right)$. Note: This figure shows the kernel density of $\sqrt{T}\left(\widehat{\rho}_{z}-\rho_{z}\right)$ for four estimators which apply the same transformation (QD, HP, FD, HD) to data and model series. Kernel densities are shown for three sample sizes $T=150$, 300, and 2000. Parameter $\rho_{z}$ is the persistence of technology shocks.
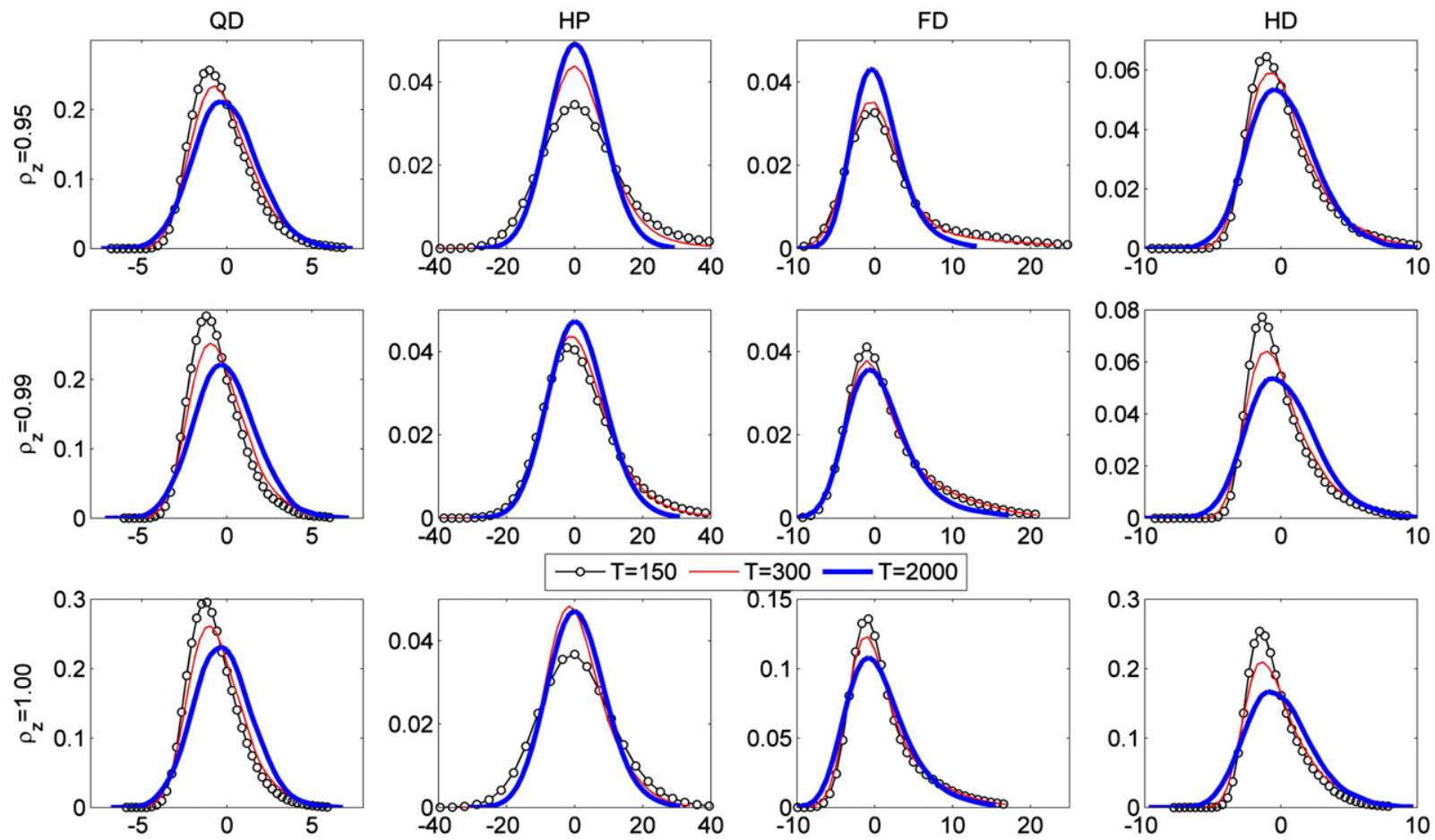

Fig. 4. Kernel density of simulated $\sqrt{T}\left(\widehat{\sigma}_{z}-\sigma_{z}\right)$. Note: This figure shows the kernel density of $\sqrt{T}\left(\widehat{\sigma}_{z}-\sigma_{z}\right)$ for four estimators which apply the same transformation (QD, HP, FD, HD) to data and model series. Kernel densities are shown for three sample sizes $T=150$, 300, and 2000. Parameter $\sigma_{z}$ is the standard deviation of technology shocks. 
Figs. 2-4 present the kernel density of the normalized estimator (i.e. $\sqrt{T}(\widehat{\Theta}-\Theta)$ ) for sample sizes of $T=150$ and 300 . Results are also reported for $T=2000$ to study the asymptotic properties of the estimators. Approximate normality of $\widehat{\rho}_{z}$ when $\rho_{z}$ is close to one, is totally unexpected, given that the literature on integrated regressors prepared us to expect super consistent estimators with Dickey-Fuller type distributions that are skewed. Instead, all densities are bell-shaped and symmetric for all $\rho_{z} \leq 1$ with no apparent discontinuity as we increase $\rho_{z}$ to one. The normal approximation is not perfect in small samples, suggesting that some size distortion will occur if one uses the $t$ statistic for inference. In unreported results, $t$-statistics constructed using Newey-West standard errors have rejection rates greater than the nominal size for all estimators except the HP, which can be undersized. For example, the rejection rate of the QD estimator for the two-sided $t$ test of $\rho_{z}$ at the true value of 1 is 0.055 when $T=200$ while for testing $\alpha$ at the true value of 0.33 , the rejection rate is 0.21 . This is larger than the nominal size of 0.05 . As the sample size increases, the actual size gets closer (and eventually converges) to the nominal rates. For example, at $T=1000$ for QD, the two-sided $t$-test of $\rho_{z}=1$ has a rejection rate of 0.05 , while the $t$ test for $\alpha=0.33$ is 0.10 . The QD and HD generally have better size than the FD and the HP. The finite sample size distortion seems to be a general problem with covariance structure estimators and not specific to the considered estimators. Burnside and Eichenbaum (1996) reported similar results in covariance structure estimation with many overidentifying restrictions, also using the Newey-West estimator of the variance of moments.

\subsection{Variations to the baseline model}

In response to the finding in Cogley and Nason (1995b) that the basic real business cycle model has weak internal propagation, researchers often augment the basic model to strengthen the propagation and to better fit the data at

Table 3

Augmented versions of the neoclassical growth model.

\begin{tabular}{|c|c|c|c|c|c|c|c|c|c|}
\hline$\rho_{z}$ & $\begin{array}{l}\text { Data filter } \\
\text { Model filer }\end{array}$ & $\begin{array}{l}\text { QD } \\
\text { QD } \\
\text { (1) }\end{array}$ & $\begin{array}{l}\text { HD } \\
\text { HD } \\
(2)\end{array}$ & $\begin{array}{l}\text { FD } \\
\text { FD } \\
\text { (3) }\end{array}$ & $\begin{array}{l}\text { HP } \\
\text { HP } \\
(4)\end{array}$ & $\begin{array}{l}\text { LT } \\
\text { LT } \\
\text { (5) }\end{array}$ & $\begin{array}{l}\mathrm{FD}_{1} \\
\mathrm{FD}_{1} \\
(6)\end{array}$ & $\begin{array}{l}\text { HP } \\
\text { LT } \\
\text { (7) }\end{array}$ & $\begin{array}{l}\mathrm{HP} \\
z_{t} \\
(8)\end{array}$ \\
\hline \multicolumn{10}{|c|}{ Panel A: serially correlated growth rate in technology } \\
\hline & & & & Estim & $\kappa=0$ & & & & \\
\hline \multirow[t]{2}{*}{0.95} & Mean & -0.010 & -0.001 & 0.001 & -0.019 & -0.180 & -0.100 & -0.224 & -0.369 \\
\hline & St.dev. & 0.063 & 0.058 & 0.050 & 0.160 & 0.165 & 0.035 & 0.038 & 0.106 \\
\hline \multirow[t]{2}{*}{0.99} & Mean & -0.014 & -0.003 & 0.002 & -0.016 & -0.498 & -0.021 & -0.255 & -0.429 \\
\hline & St.dev. & 0.044 & 0.046 & 0.041 & 0.155 & 0.088 & 0.030 & 0.038 & 0.070 \\
\hline \multirow[t]{2}{*}{1.00} & Mean & -0.014 & -0.003 & 0.003 & -0.020 & -0.600 & -0.002 & -0.256 & -0.446 \\
\hline & St.dev. & 0.038 & 0.039 & 0.035 & 0.161 & 0.029 & 0.028 & 0.038 & 0.058 \\
\hline \multicolumn{10}{|c|}{ Panel B: habit formation in consumption } \\
\hline \multirow{3}{*}{0.95} & & & & Estim & $\phi=0$ & & & & \\
\hline & Mean & 0.020 & 0.008 & 0.006 & -0.014 & -0.410 & -0.086 & 0.193 & 0.679 \\
\hline & St.dev. & 0.075 & 0.073 & 0.071 & 0.255 & 0.339 & 0.066 & 0.382 & 0.074 \\
\hline \multirow[t]{2}{*}{0.99} & Mean & 0.019 & 0.009 & 0.008 & 0.023 & -0.647 & -0.018 & 0.495 & 0.637 \\
\hline & St.dev. & 0.070 & 0.074 & 0.069 & 0.168 & 0.241 & 0.083 & 0.400 & 0.070 \\
\hline \multirow[t]{2}{*}{1.00} & Mean & 0.018 & 0.023 & 0.011 & 0.025 & -0.702 & 0.011 & 0.603 & 0.622 \\
\hline & St.dev. & 0.067 & 0.087 & 0.076 & 0.156 & 0.174 & 0.095 & 0.373 & 0.068 \\
\hline
\end{tabular}

Panel C: preference shocks $q_{t}$

\begin{tabular}{|c|c|c|c|c|c|c|c|c|c|}
\hline \multicolumn{10}{|c|}{ Estimate of $\alpha=0.33$} \\
\hline & $\sigma_{q}=0.5$ & & & & & & & & \\
\hline \multirow[t]{2}{*}{0.95} & Mean & 0.344 & 0.335 & 0.361 & 0.329 & 0.465 & 0.299 & 0.591 & 0.337 \\
\hline & St.dev. & 0.040 & 0.025 & 0.080 & 0.075 & 0.126 & 0.043 & 0.037 & 0.137 \\
\hline \multirow[t]{2}{*}{1.00} & Mean & 0.353 & 0.342 & 0.352 & 0.335 & 0.508 & 0.352 & 0.665 & 0.485 \\
\hline & $\begin{array}{l}\text { St.dev. } \\
\sigma_{q}=1.0\end{array}$ & 0.052 & 0.034 & 0.067 & 0.072 & 0.357 & 0.063 & 0.050 & 0.265 \\
\hline \multirow[t]{2}{*}{0.95} & Mean & 0.339 & 0.341 & 0.349 & 0.331 & 0.431 & 0.316 & 0.504 & 0.344 \\
\hline & St.dev. & 0.023 & 0.030 & 0.049 & 0.060 & 0.103 & 0.022 & 0.031 & 0.022 \\
\hline \multirow[t]{2}{*}{1.00} & Mean & 0.347 & 0.347 & 0.357 & 0.340 & 0.611 & 0.341 & 0.529 & 0.364 \\
\hline & $\begin{array}{l}\text { St.dev. } \\
\sigma_{q}=1.5\end{array}$ & 0.028 & 0.034 & 0.050 & 0.055 & 0.257 & 0.025 & 0.036 & 0.023 \\
\hline \multirow[t]{2}{*}{0.95} & Mean & 0.338 & 0.343 & 0.349 & 0.333 & 0.399 & 0.326 & 0.469 & 0.378 \\
\hline & St.dev. & 0.021 & 0.030 & 0.042 & 0.053 & 0.078 & 0.017 & 0.020 & 0.024 \\
\hline \multirow[t]{2}{*}{1.00} & Mean & 0.341 & 0.346 & 0.353 & 0.338 & 0.515 & 0.338 & 0.477 & 0.391 \\
\hline & St.dev. & 0.020 & 0.029 & 0.038 & 0.049 & 0.203 & 0.017 & 0.021 & 0.023 \\
\hline
\end{tabular}

Note: Panels A and B: $\rho_{z}$ and $\kappa$ or $\phi$ are estimated; $\alpha=0.33$ and $\sigma_{z}=1$ are fixed. Panel C: five parameters are estimated $\left(\alpha, \rho_{z}, \rho_{q}, \sigma_{z}, \sigma_{q}\right)$. The number of simulations is 2000. Sample size is T=200. LT is linear detrending, HP is Hodrick-Prescott filter, FD is first differencing, FD $_{1}$ is first differencing with the restriction that $\rho_{z}=1$, QD is quasi-differencing, HD is hybrid differencing, $z_{t}$ is detrending by the level of technology. 
business cycle frequencies. One consideration is to introduce serial correlation in the growth rate of shocks to technology by assuming $u_{t}=\left(\rho_{z}+\kappa\right) u_{t-1}-\kappa \rho_{z} u_{t-2}+e_{t}^{z}$. This specification generates serial correlation of $\kappa$ in the growth rate of technology when $\rho_{z} \approx 1$. The baseline model corresponds to $\kappa=0$. When data are simulated with $\kappa=0$ and $\kappa$ is estimated freely, the QD, HD, FD, and HP correctly find that $\kappa=0$ (Table 3, Panel A).

Habit in consumption is another popular way to introduce greater persistence in business cycle models. Consider the utility function: $\ln \left(C_{t}-\phi C_{t-1}\right)-\theta L_{t}$ where $\phi$ measures the degree of habit in consumption. Data are generated with $\phi$ set to zero. When $\phi$ is freely estimated along with other parameters, the robust estimators again find $\widehat{\phi}$ to be numerically small and not statistically different from zero for all values of $\rho_{z}$ (Table 3, Panel B).

A third variation to the baseline model is a preference shock $Q_{t}$ such that the utility is $\ln C_{t}-\theta L_{t} / Q_{t}$ where $q_{t}=\ln Q_{t}=\rho_{q} q_{t-1}+e_{t}^{q}$ and $e_{t}^{q} \sim i i d\left(0, \sigma_{q}^{2}\right)$. In the simulations, $\rho_{q}=0.8$ (so that the preference shock is stationary) and $\sigma_{q}=(0.5,1.0,1.5)$. To conserve space, Panel $C$ in Table 3 reports only estimates for $\alpha$. Consistent with the results thus far, the HP estimates have the largest variability although the difference with other estimators is not as large as it was in the baseline model. Note that as $\sigma_{q}$ increases, the difference across methods shrinks while the precision for all estimators improves.

A recurrent result is that the HP estimates have the largest variability and is computationally most intensive. Burnside (1998) reports that the HP filter removes variation potentially informative about the structural parameters but that the HP filtered model and data series still have sufficient variability to discriminate competing theories of business cycles. One possibility for the results reported here is that the HP filters out more low frequency variation than other filters, and the parameters $\phi$ and $\kappa$ are identified from these frequencies. Another possibility is that the HP implicitly uses many more estimated autocovariances (recall that the inverse Fourier transform is applied to many autocovariances). This extensive use of sample autocovariances can also introduce variability to the estimator.

\section{Non-robust estimators and a model with multiple rigidities}

This section reports results for the non-robust estimators to illustrate how treatment of trends can bring about misleading conclusions about the propagating mechanism of shocks. In addition to the basic stochastic growth model, the estimators are also compared for a model with many more endogenous variables.

\subsection{Alternative detrending procedures}

Up to this point, the considered approaches apply the same transformation to the data and the model variables. Much has been written about the effects of filtering on business cycle facts. King and Rebelo (1993) and Canova (1998) showed that the HP filtered data are qualitatively different from the raw data. Canova (1998) showed that the stylized facts of business cycles are sensitive to the filter used to remove the trending components. Gregory and Smith (1996) used a calibrated business cycle model to investigate what type of trend can produce a cyclical component in the data that is similar to the cyclical component in the model. Although these authors did not estimate a DSGE model on filtered data, they hinted that the parameter estimates can be adversely affected by filtering.

To investigate the consequences of using different and/or inappropriate filters, four combinations are considered: (A) the autocovariances are computed for linearly detrended model and data series; (B) the autocovariances are computed for the first differenced model and data series with imposed $\rho_{z}=1 ;(\mathrm{C})$ the sample autocovariances are computed for HP filtered data but the model autocovariances are computed for the linearly detrended variables; (D) the sample autocovariances are computed for HP filtered data while the model autocovariances are computed for series normalized by the level of technology, i.e., $m_{t}-z_{t}$ where $z_{t}$ is the level of technology.

Each combination has been used in the literature (see e.g. Table 1). (A) and (B) are aimed to show the effects of imposing incorrect assumptions about trends. (C) and (D) illustrate the consequences when different trends are applied to the model and the data. ${ }^{6}$ The results for the basic stochastic growth model are reported in Table 2. For (A), which is reported in column (5), the parameter estimates are slightly biased when $\rho_{z}=0.95$. As $\rho_{z}$ increases, the estimates are strongly biased. This shows that when $\rho_{z}$ is close to unity yet stationary, assuming trend stationarity still yields imprecise estimates. At $\rho_{z}=1$, the mean of $\widehat{\rho}_{z}$ is 0.694 (instead of 1 ), the mean of $\widehat{\alpha}$ is approximately 0.905 (instead of 0.33 ), the mean of $\widehat{\sigma}_{z}$ is 19.8 (instead of 1 ). The case of $\rho_{z} \leq 1$ is empirically relevant because macroeconomic data are highly persistent and well approximated by unit root processes. These results show that linear detrending of nearly integrated data in non-linear estimation can lead to biased estimates of the structural parameters, reminiscent of the univariate finding of Nelson and Kang (1981) that projecting a series with a unit root on time trend can lead to spurious cycles.

Turning to (B) in column (6) of Table 2, the estimates are fairly precise when $\rho_{z}$ is indeed equal to one, but as $\rho_{z}$ departs from one, the estimates get increasingly biased. Hence imposing a stochastic trend when the data generating process is trend stationary can lead to seriously distorted estimates. Results for combination (C) are reported in column (7) of Table 2.

\footnotetext{
${ }^{6}$ As a general observation, the starting values are very important for non-robust methods as the optimization routines can get stuck in local optima. With the robust estimators, the converged estimates do not change as the optimization starts from values other than the true parameters, though the search for global minimum was often long.
} 
The estimates of $\rho_{z}$ are downward biased while $\widehat{\alpha}$ and $\widehat{\sigma}_{z}$ are upward biased. Taken at face value, these estimates suggest a significant role for capital as a mechanism for propagating shocks in the model.

Results for (D) are reported in column (8) of Table 2. Here, the estimates of $\alpha$ often hit the boundary of the permissible parameter space while estimates of $\sigma_{z}$ are close to zero. The reason is that when $z_{t}$ has a unit root, shocks to $m_{t}-z_{t}$ are transitory and consumption adjusts quickly to the permanent technology shock. But the HP filtered data are serially correlated. Thus, the estimator is forced to produce parameter values that can generate strong serial correlation in the model variables. Results for (C) and (D) are consistent with the findings of Cogley and Nason (1995a), King and Rebelo (1993) and Harvey and Jaeger (1993). These papers suggest that the HP filter changes not only the persistence of the series but also the relative volatility and serial correlation of the series. This translates into biased estimates of all parameters because the estimator is forced to match the serial correlation of the filtered data.

Clearly, large estimated values of $\alpha$ will alert the researcher that the model is likely misspecified. Suppose the researcher allows for serially correlated shocks in technology growth by estimating $\kappa$ freely. Panel A in Table 3 shows that the non-robust methods now yield estimates of $\alpha$ around $0.4-0.5$, which seem more plausible than when $\kappa$ was assumed zero. However, these estimates are achieved by having $\widehat{\kappa}$ strongly negative and statistically significant when the true value of $\kappa$ is zero. Suppose now the researcher modifies the model by allowing for habits in consumption. Evidently, the estimated habit formation parameter $\phi$ is sensitive to which non-robust estimator is used. In particular, (A) has a strong downward bias, while (B) produces a negative bias in $\widehat{\phi}$ when $\rho_{z}$ departs from one. On the other hand, (C) and (D) have a strong upward bias. With either modification, the fit of the misspecified models improves relative to the correctly specified model. However, these modifications should not have been undertaken as they do not exist in the data generating process. These examples indicate how the treatment of trends can mislead the researcher to augment correctly specified models with spurious propagation mechanisms to match the moments of the data.

Results for the model with an additional labor supply shock are reported in Table 3, Panel C. The estimates continue to be biased although the biases tend to be smaller than in the baseline model with a single persistent shock. In general, a

Table 4

Smets and Wouters (2007) model.

\begin{tabular}{|c|c|c|c|c|c|c|c|c|c|}
\hline$\rho_{z}$ & $\begin{array}{l}\text { Data filter } \\
\text { Model filer }\end{array}$ & $\begin{array}{l}\text { QD } \\
\text { QD } \\
(1)\end{array}$ & $\begin{array}{l}\text { HD } \\
\text { HD } \\
(2)\end{array}$ & $\begin{array}{l}\text { FD } \\
\text { FD } \\
(3)\end{array}$ & $\begin{array}{l}\text { HP } \\
\text { HP } \\
(4)\end{array}$ & $\begin{array}{l}\text { LT } \\
\text { LT } \\
(5)\end{array}$ & $\begin{array}{l}\mathrm{FD}_{1} \\
\mathrm{FD}_{1} \\
(6)\end{array}$ & $\begin{array}{l}\text { HP } \\
\text { LT } \\
(7)\end{array}$ & $\begin{array}{l}\text { HP } \\
z t \\
(8)\end{array}$ \\
\hline \multicolumn{10}{|c|}{ Estimate of persistence in technology shocks $\rho_{z}$} \\
\hline \multirow[t]{2}{*}{0.95} & Mean & 0.965 & 0.967 & 0.962 & 0.945 & 0.864 & 1.000 & -0.100 & 1.000 \\
\hline & St.dev. & 0.038 & 0.037 & 0.044 & 0.137 & 0.142 & & 0.157 & \\
\hline \multirow[t]{2}{*}{0.99} & Mean & 0.986 & 0.984 & 0.986 & 0.967 & 0.836 & 1.000 & -0.114 & 1.000 \\
\hline & St.dev. & 0.027 & 0.027 & 0.028 & 0.123 & 0.227 & & 0.090 & \\
\hline \multirow[t]{2}{*}{1.00} & Mean & 0.990 & 0.989 & 0.993 & 0.971 & 0.744 & 1.000 & -0.123 & 1.000 \\
\hline & St.dev. & 0.027 & 0.026 & 0.025 & 0.123 & 0.305 & & 0.075 & \\
\hline \multicolumn{10}{|c|}{ Estimate of investment adjustment cost $\phi=5.48$} \\
\hline \multirow[t]{2}{*}{0.95} & Mean & 5.057 & 5.381 & 5.227 & 5.066 & 3.932 & 4.700 & 4.447 & 9.818 \\
\hline & St.dev. & 2.236 & 2.548 & 2.306 & 3.354 & 1.917 & 2.487 & 0.265 & 0.609 \\
\hline \multirow[t]{2}{*}{0.99} & Mean & 5.432 & 5.563 & 5.373 & 5.095 & 5.595 & 5.236 & 4.366 & 9.662 \\
\hline & St.dev. & 2.321 & 2.463 & 2.404 & 3.012 & 2.647 & 2.794 & 0.257 & 0.588 \\
\hline \multirow[t]{2}{*}{1.00} & Mean & 5.863 & 6.253 & 6.014 & 5.617 & 6.173 & 6.049 & 4.377 & 9.541 \\
\hline & St.dev. & 2.375 & 2.775 & 2.781 & 3.279 & 2.983 & 3.046 & 0.230 & 0.548 \\
\hline \multicolumn{10}{|c|}{ Estimate of habit formation $\lambda=0.71$} \\
\hline \multirow[t]{2}{*}{0.95} & Mean & 0.725 & 0.730 & 0.749 & 0.753 & 0.730 & 0.864 & 3.932 & 0.673 \\
\hline & St.dev. & 0.057 & 0.063 & 0.062 & 0.049 & 0.063 & 0.142 & 1.917 & 0.134 \\
\hline \multirow[t]{2}{*}{0.99} & Mean & 0.699 & 0.718 & 0.719 & 0.718 & 0.543 & 0.744 & 0.908 & 0.941 \\
\hline & St.dev. & 0.056 & 0.053 & 0.062 & 0.134 & 0.177 & 0.053 & 0.033 & 0.006 \\
\hline \multirow[t]{2}{*}{1.00} & Mean & 0.686 & 0.711 & 0.716 & 0.709 & 0.470 & 0.731 & 0.912 & 0.940 \\
\hline & St.dev. & 0.056 & 0.055 & 0.064 & 0.145 & 0.261 & 0.057 & 0.028 & 0.005 \\
\hline \multicolumn{10}{|c|}{ Estimate of wage adjustment probability $\xi_{w}=0.73$} \\
\hline \multirow[t]{2}{*}{0.95} & Mean & 0.704 & 0.730 & 0.734 & 0.686 & 0.657 & 0.759 & 0.484 & 0.220 \\
\hline & St.dev. & 0.073 & 0.063 & 0.075 & 0.117 & 0.105 & 0.077 & 0.085 & 0.019 \\
\hline \multirow[t]{2}{*}{0.99} & Mean & 0.686 & 0.704 & 0.709 & 0.659 & 0.530 & 0.718 & 0.458 & 0.213 \\
\hline & St.dev. & 0.081 & 0.065 & 0.079 & 0.125 & 0.214 & 0.084 & 0.078 & 0.016 \\
\hline \multirow[t]{2}{*}{1.00} & Mean & 0.673 & 0.697 & 0.700 & 0.641 & 0.457 & 0.700 & 0.444 & 0.210 \\
\hline & St.dev. & 0.092 & 0.068 & 0.083 & 0.138 & 0.262 & 0.091 & 0.072 & 0.015 \\
\hline
\end{tabular}

Note: The number of simulations is 2000 . Sample size is $T=150$. LT is linear detrending, HP is Hodrick-Prescott filter, FD is first differencing, FD ${ }_{1}$ is first differencing with the restriction that $\rho_{z}=1, \mathrm{QD}$ is quasi-differencing, HD is hybrid differencing, $z_{t}$ is detrending by the level of technology. 

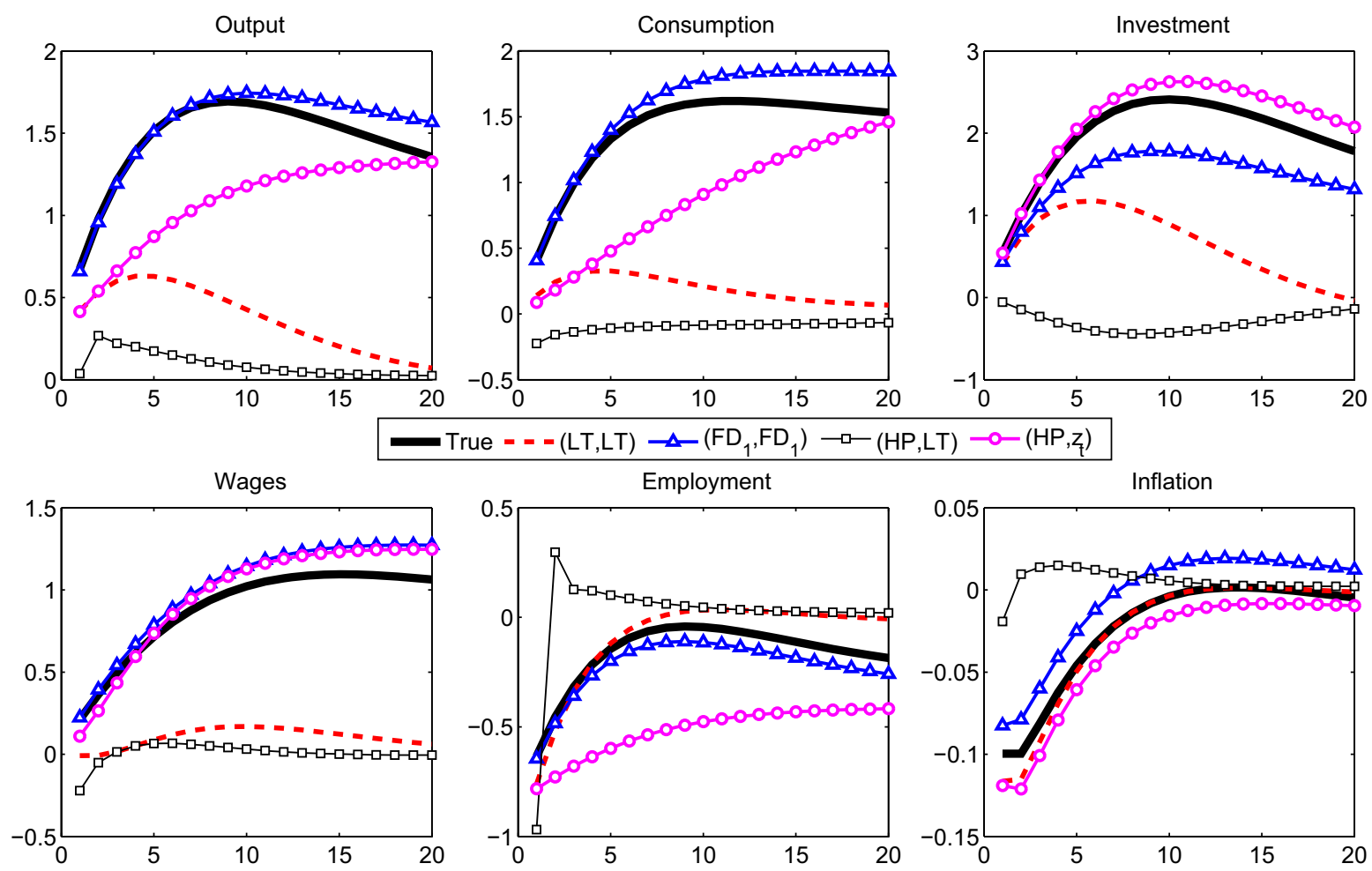

Fig. 5. Estimated impulse responses functions to a technology shock in Smets and Wouters (2007) model. Note: This figure plots impulse response functions based on parameter estimates obtained from estimators applying different filters to model and data series when the data are generated by the Smets and Wouters (2007) model. LT is projection on a linear time trend. $\mathrm{FD}_{1}$ is first differencing. HP is the Hodrick-Prescott filter. The shock is a 1 percent increase in the level of technology. Persistence of technology shock is $\rho_{z}=0.99$. See supplemental material for other impulse responses.

smaller $\rho_{z}$ and a larger $\sigma_{q}$ lead to smaller biases. In some cases, one finds $\widehat{\sigma}_{q}>\widehat{\sigma}_{z}$, so that the researcher may be tempted to conclude that preference shocks have larger volatility than shocks to technology while the opposite is true.

\subsection{The Smets and Wouters model}

Although the baseline model is an illuminating laboratory to evaluate how the estimators perform, it is overly simplistic. To assess the properties of the estimators in a more realistic setting, consider the model of Smets and Wouters (2007) (henceforth SW). Treating SW's estimates for the post-1982 sample as the true parameter values, series of size $T=150$ are generated and the estimators are applied to the generated series. To separate identification issues from issues related to the treatment of trends, only four parameters are estimated: persistence of technology shocks $\rho_{z}$ whose true value varies across simulations; investment adjustment cost $\phi$ whose true value is 5.48; external habit formation in consumption $\lambda$ whose true value is 0.71 ; and Calvo's probability of wage adjustment $\xi_{w}$ whose true value is 0.73 .

The results are reported in Table 4. All robust methods yield precise estimates of the parameters. Although the HP continues to be less precise, the difference with the other three robust estimators is smaller than in the baseline model. A similar feature was observed when the two-shock and one-shock neoclassical growth models were compared. These differences between the baseline and the more complicated models can occur for several reasons. First, in larger models with many other structural shocks, technology shock explains only a fraction of variation in key macroeconomic variables. The HP estimator may simply need more shocks to identify the parameters. Second, bigger models impose many more cross equation restrictions that may improve the efficiency of some estimators more than others. The general observation, however, is that the proposed robust estimators perform reasonably well for all values of $\rho_{z}$ in simple and more complex models.

In contrast, the non-robust estimators (A) through (D) have dramatic biases in all four parameters being estimated when (i) the filter used for the model and the data are different, when (ii) the assumed trends are different from trends in the data generating process, or when (iii) the data are stationary but highly persistent. Obviously, the impulse responses (and other analyses related to the role of rigidities in amplification and propagation of shocks in business cycle models) based on these biased estimates of the structural parameters will be misleading. As an illustration, Fig. 5 highlights the difference between the true response of key macroeconomic variables to a technology shock in the SW model and the responses based on parameter estimates from approaches (A) through (D). For instance, consider the response of 
consumption. Estimates from approaches (A) and (C) imply grossly understated responses. Estimates from approach (D) suggest a considerably more delayed consumption response than the true one. The consumption response implied by approach (B) is qualitatively similar to the true response, but the responses are noticeably different quantitatively especially when $\rho_{z}$ is further away from one.

\section{Extensions and implementation issues}

This section discusses several practical issues and extensions pertaining to the robust estimators.

\subsection{Multiple shocks}

The reduced form solution (1) can be easily generalized to other models and takes the form

$$
\begin{aligned}
& \widehat{m}_{t}=\Pi \widehat{m}_{t-1}+B u_{t} \\
& u_{t}=\rho u_{t-1}+S e_{t}
\end{aligned}
$$

where $u_{t}$ is now a vector of exogenous forcing variables, $e_{t}$ is a vector of innovations in $u_{t}$, and the matrices $\Pi, B, S, \rho$ are of conformable sizes.

Suppose there are $J$ univariate shock processes, each characterized by

$$
\left(1-\rho_{j} L\right) u_{j t}=e_{j t}, \quad j=1, \ldots, J
$$

where some $J^{*}$ of the $\rho_{j}$ may be on the unit circle. Define

$$
\Delta^{\rho}(L)=\prod_{j=1}^{J^{*}}\left(1-\rho_{j} L\right)
$$

Now the quasi-differencing operator is the product of the $J^{*}$ polynomials in lag operator. Once the model is solved to arrive at (3), one can compute moments for $\Delta^{\rho}(L) \hat{m}_{t}$. Whether none, one, or more shocks are permanent, the autocovariances of the transformed variables are well defined. For example, if one knows that shocks to tastes dissipate quickly while technology shocks $z_{t}$ are highly persistent, one can still use $\left(1-\rho_{z} L\right)$ as $\Delta^{\rho}$.

\subsection{Likelihood estimation}

As likelihood and Bayesian estimation is commonly used in the DSGE literature, one may wonder how the ideas considered in this paper can be implemented in likelihood based estimation. Suppose one can write the model in a state space form which involves using the measurement equations to establish a strict correspondence between the detrended series in the model and in the data. Then one can derive the likelihood which makes maximum likelihood (MLE) and Bayesian estimation possible.

As an example, consider the model given in (3). The measurement equation corresponding to the FD estimator is

$$
x_{t}=H s_{t}=\left[\begin{array}{lll}
\Psi & -\Psi & 0
\end{array}\right] s_{t}
$$

where $x_{t}$ is the vector of filtered variable, $\Psi$ is the selection matrix, and $s_{t}^{\prime}=\left(\widehat{m}_{t}, \widehat{m}_{t-1}, u_{t}\right)$ is the state vector. The corresponding transition equation is

$$
\left[\begin{array}{c}
\widehat{m}_{t} \\
\widehat{m}_{t-1} \\
u_{t}
\end{array}\right]=\left[\begin{array}{ccc}
\Pi & 0 & B \rho \\
I & 0 & 0 \\
0 & 0 & \rho
\end{array}\right]\left[\begin{array}{c}
\widehat{m}_{t-1} \\
\widehat{m}_{t-2} \\
u_{t-1}
\end{array}\right]+\left[\begin{array}{c}
B S \\
0 \\
S
\end{array}\right] e_{t}
$$

or

$$
s_{t}=\Pi^{*} s_{t-1}+B^{*} e_{t}
$$

with $e_{t} \sim$ i.i.d. $(0, \Sigma)$. The measured variable $x_{t}$ is stationary irrespective of whether $\widehat{m}_{t}$ has stochastic or deterministic trends. For the $\mathrm{QD}^{0}$ estimator, $H=\left[\begin{array}{l}\Psi-\rho \Psi \\ 0\end{array}\right]$. As with all quasi-differencing estimators, the treatment of initial condition is important especially when there is strong persistence. In simulations with the first observation held fixed, the MLE version of the FD gives precise estimates, but the $t$ statistics are less well approximated by the normal distribution compared to MM-FD (see supplementary material).

For the other three estimators, the extension to MLE is either not possible or not practical. For MLE-HP, one would need to write out the entire data density of the HP filtered data, and the Jacobian transformation from the unfiltered to filtered data involves an infinite dimensional matrix. For the QD estimator, recall that the autocovariances are normalized by the variance. By analogy, MLE-QD would require modifying the score vector. Although such modification is possible in theory, it is not straightforward to implement. For the HD estimator, the MLE implementation is cumbersome because HD exploits 
covariances of variables computed with different filters. The difference between the MM and MLE really boils down to a choice of moments, and the MM is more straightforward to implement.

\subsection{Computation}

Moments of the filtered model variables can be computed analytically or by using simulations. We use the analytical moments whenever possible since it tends to be much faster than simulations and it does not have simulation errors. Although there are a variety of methods for analytical calculations, a method that is especially attractive for large models is to combine the measurement equation $x_{t}=H s_{t}$ and the state equation $s_{t}=\Pi^{*} s_{t-1}+B^{*} e_{t}$ to obtain

$$
\left[\begin{array}{c}
x_{t} \\
s_{t}
\end{array}\right]=\left(\begin{array}{cc}
0 & H \Pi^{*} \\
0 & \Pi^{*}
\end{array}\right)\left[\begin{array}{c}
x_{t-1} \\
s_{t-1}
\end{array}\right]+\left(\begin{array}{c}
H B^{*} \\
B^{*}
\end{array}\right) e_{t}
$$

Let $w_{t}^{\prime}=\left(x_{t}^{\prime}, s_{t}^{\prime}\right)$ so that

$$
w_{t}=D_{0} w_{t-1}+D_{1} e_{t}
$$

The variance matrix $\Omega_{w}(0)=E\left(w_{t} w_{t}^{\prime}\right)$ can now be computed by iterating the equation

$$
\Omega_{w}^{(i)}(0)=D_{0} \Omega_{w}^{(i-1)}(0) D_{0}^{\prime}+D_{1} \Sigma D_{1}^{\prime}
$$

until convergence. The autocovariance matrices can then be computed as $\Omega_{w}(j)=D_{0}^{k} \Omega_{w}(0)$. Since one is only interested in computing the moments of variables in the measurement vector $x_{t}$, one can iterate Eq. (6) until the block that corresponds to $x_{t}$ converges, i.e. $\left\|\Omega_{x}^{(i)}(0)-\Omega_{x}^{(i-1)}(0)\right\|<\varepsilon$.

To compute the moments of the HP filtered data, observe that the HP filtered series can alternatively be obtained as follows:

$$
H P(L) d_{t}=H P^{+}(L) \Delta d_{t}=\frac{\lambda(1-L)\left(1-L^{-1}\right)^{2}}{1+\lambda(1-L)^{2}\left(1-L^{-1}\right)^{2}} \Delta d_{t}
$$

In practice, using $H P^{+}(\mathrm{L})$ and the autocovariances for $\Delta d_{t}$ and $\Delta \widehat{m}_{t}$ tends to give more stable results when $\rho_{z}$ is close to one. It is possible to speed up estimation based on HP filtered series by using a smaller number of leads and lags at the cost of larger approximation errors. ${ }^{7}$

Finally, a note on the treatment of stationary variables is in order. Recall that in the stochastic growth model, $m_{t}^{*}=(\bar{g} t, \bar{g} t, 0)$ when $\left|\rho_{z}\right|<1$ and $m_{t}^{*}=\left(u_{t}+\bar{g} t, u_{t}+\bar{g} t, 0\right)$ when $\left|\rho_{z}\right|=1$, where the third component of $m_{t}^{*}$ is the trend for labor supply, $l_{t}$. Since $l_{t}$ has no deterministic or stochastic trend component, the autocovariances are computed for $l_{t}$ and not $\widehat{l}_{t}$, though the results do not change materially if the filtered series were used. In general, if the $j$-th component of $m_{t}^{*}$ is zero, it is understood that the autocovariances are computed for the level of the variable both in the model and in the data. An alternative is to deal with these non-trending variables through the measurement equation. Then some variables can be quasi-differenced or first-differenced, while others require no transformation.

\section{Concluding remarks}

A realistic situation encountered with estimation of DSGE model is that (a) the data are trending; (b) deviations from the trend are persistent; (c) the researcher does not know whether the data generating process is difference or trend stationary. This paper shows that the treatment of trends can significantly affect the parameter estimates of DSGE models and propose several robust approaches that produce precise estimates without the researcher having to take a stand of trend specification. The key is to apply the same filter to the data and the model variables to yield well-defined moments for the estimation of the structural parameters. Several filters can be used in methods of moments estimation. These estimators have approximately normal finite sample distributions. Undoubtedly, the estimators require further scrutiny and can be improved in various dimensions. ${ }^{8}$ The present analysis is a first step in the sparse literature on non-linear estimation when the data are highly persistent.

\section{Appendix A. Supplementary data}

Supplementary data associated with this article can be found in the online version at doi:10.1016/j.jmoneco.2010.02.008.

\footnotetext{
${ }^{7}$ A simulation procedure can also be considered. For each $\Theta$, the model is used to generate $j=1, \ldots, R$ samples of size $T$ and the moments are computed. Averaging over $j$ gives $\omega_{H p}^{m}$. This procedure is computationally more intensive and the results are similar to the one considered here.

${ }^{8}$ For example, one can use the bootstrap developed for covariance structures in Horowitz (1998) to correct for small-sample biases. One might also consider a model-based instead of a data-based weighting matrix computed. Finally, one may use simulation based estimators, see Coibion and Gorodnichenko (2010) for an example.
} 


\section{References}

Abowd, J.M., Card, D., 1989. On the covariance structure of earnings and hours changes. Econometrica 57 (2), $411-445$.

Altig, D., Christiano, L., Eichenbaum, M., Linde, J., 2004. Firm-specific capital, nominal rigidities, and the business cycle, FRB Cleveland WP 2004-16. Altonji, J., Segal, L., 1996. Small-sample bias in GMM estimation of covariance structures. Journal of Business and Economic Statistics 14 (3), 353-366. Altug, S., 1989. Time-to-build and aggregate fluctuations: some new evidence. International Economic Review 30, 889-920.

Anderson, G., Moore, G., 1985. A linear algebraic procedure for solving linear perfect foresight models. Economic Letters 17 (3), $247-252$.

Baxter, M., King, R., 1999. Measuring business cycles: approximate bandpass filters for economic time series. Review of Economics and Statistics 81 (4), $575-593$.

Blanchard, O., Kahn, C., 1980. The solution of linear difference models under rational expectations. Econometrica 48, 1305-1313.

Bouakez, H., Cardia, E., Ruge-Murcia, F.J., 2005. Habit formation and the persistence of monetary shocks. Journal of Monetary Economics 52, 1073-1088. Burnside, C., 1998. Detrending and business cycle facts: a comment. Journal of Monetary Economics 41, 513-532.

Burnside, C., Eichenbaum, M., 1996. Small-sample properties of GMM-based Wald tests. Journal of Business and Economic Statistics 14, 294-308.

Burnside, C., Eichenbaum, M., Rebelo, S., 1993. Labor hoarding and the business cycle. Journal of Political Economy 101, $245-273$.

Canova, F., 1998. Detrending and business cycle facts. Journal of Monetary Economics 41, 475-512.

Canova, F., 2008. Estimating DSGE models with unfiltered data. Mimeo.

Canova, F., Ferroni, F., 2008. Multiple filtering devices for the estimation of cyclical DSGE models. Mimeo.

Canova, F., Sala, L., 2009. Back to square one: identification issues in DSGE models. Journal of Monetary Economics 56, $431-449$.

Christiano, L., den Haan, W., 1996. Small-sample properties of GMM for business cycle analysis. Journal of Business and Economic Statistics 14, 309-327.

Christiano, L., Eichenbaum, M., 1992. Current real-business-cycle theories and aggregate labor-market fluctuations. American Economic Review 82, 430-450.

Christiano, L.J., Eichenbaum, M., Evans, C.L., 2005. Nominal rigidities and the dynamic effects of a shock to monetary policy. Journal of Political Economy 113 (1), 1-45.

Clarida, R., Gali, J., Gertler, M., 2000. Monetary policy rules and macroeconomic stability: evidence and some theory. Quarterly Journal of Economics 115 $147-180$.

Cogley, T., 2001. Estimating and testing rational expectations models when the trend specification is uncertain. Journal of Economic Dynamics and Control 25, 1485-1525.

Cogley, T., Nason, J., 1995a. Effects of the Hodrick-Prescott filter on trend and difference stationary time series: implications for business cycle research. Journal of Economic Dynamics and Control 19, 253-278.

Cogley, T., Nason, J., 1995b. Output dynamics in real business cycle models. American Economic Review 85, $492-511$.

Coibion, O., Gorodnichenko, Y., 2010. Strategic interaction among heterogeneous price-setters in an estimated DSGE model. Review of Economics and Statistics, forthcoming.

Del Negro, M., Schorfheide, F., Smets, F., Wouters, R., 2007. On the fit of new Keynesian models. Journal of Business and Economic Statistics 25, 123-143.

Dib, A., 2003. An estimated Canadian DSGE model with nominal and real rigidities. Canadian Journal of Economics 36, 949-972.

Doorn, D., 2006. Consequences of Hodrick-Prescott filtering for parameter estimation in a structural model of inventory. Applied Economics 38 , $1863-1875$.

Faia, E., 2007. Finance and international business cycles. Journal of Monetary Economics 54, 1018-1034.

Fernandez-Villaverde, J., Rubio-Ramirez, J.F., 2007. Estimating macroeconomic models: a likelihood approach. Review of Economic Studies 74 (4), 1059-1087.

Fuhrer, J., 1997. The (un)importance of forward-looking behavior in price specifications. Journal of Money, Credit, and Banking 29, 338-350.

Fuhrer, J., Rudebusch, G., 2004. Estimating the Euler equation for output. Journal of Monetary Economics 51, 1133-1153.

Fukac, M., Pagan, A., 2006. Limited information estimation and evaluation of DSGE models, NCER Working Paper No. 6.

Gorodnichenko, Y., Mikusheva, A., Ng, S., 2009. A simple root-t consistent and asymptotically normal estimator for the largest autoregressive root. Mimeo. Gregory, A., Smith, G., 1996. Measuring business cycles with business-cycle models. Journal of Economic Dynamics and Control 20, $1007-1025$.

Harvey, A., Jaeger, A., 1993. Detrending, stylized factors, and the business cycle. Journal of Applied Econometrics 8, $231-247$.

Horowitz, J., 1998. Bootstrap methods for covariance structures. Journal of Human Resources 33, 39-61.

Ireland, P., 1997. A small structural quarterly model for monetary policy evaluation. Carnegie-Rochester Conference Series on Public Policy 47, 83-108.

Ireland, P., 2001. Sticky-price models of the business cycle: specification and stability. Journal of Monetary Economics 47, 3-18.

Ireland, P., 2004. Technology shocks in the new Keynesian model. Review of Economics and Statistics 86, $923-936$.

Iskrev, N., 2010. Local identication in DSGE models. Journal of Monetary Economicss 57 (2), 189-202.

Kim, J., 2000. Constructing and estimating a realistic optimizing model of monetary policy. Journal of Monetary Economics 45, 329-359.

King, R., Rebelo, S., 1993. Low frequency filtering and real business cycles. Journal of Economic Dynamics and Control 17, $207-231$.

Komunjer, I., Ng, S., 2009. Dynamic identification of DSGE models. Unpublished manuscript, Columbia University.

Kydland, F., Prescott, E., 1982. Time to build and aggregate fluctuations. Econometrica 50, 1345-1370.

Lubik, T., Schorfheide, F., 2004. Testing for indeterminacy: an application to U.S. monetary policy. American Economic Review 94, 190-217.

McGrattan, E., Rogerson, R., Wright, R., 1997. An equilibrium model of the business cycle with household production and fiscal policy. International Economic Review 38, 267-290.

Nelson, C., Kang, H., 1981. Spurious periodicity in inappropriately detrended time series. Econometrica 49, $741-751$.

Newey, W., McFadden, D., 1994. Large sample estimators and hypothesis testing. In: Engle, R., Intriligator, D. (Eds.), Handbook of Econometrics, vol. 4. North Holland, Amsterdam (Chapter 36).

Ruge-Murcia, F., 2007. Methods to estimate dynamic stochastic general equilibrium models. Journal of Economic Dynamics and Control 31, 2599-2636.

Sbordone, A., 2006. U.S. wage and price dynamics: a limited-information approach. International Journal of Central Banking 2 (3), $155-191$.

Sims, C., 2002. Solving linear rational expectations models. Computational Economics 1/2, 1-20.

Singleton, K.J., 1988. Econometric issues in the analysis of equilibrium business cycle models. Journal of Monetary Economics 21, 361-368.

Smets, F., Wouters, R., 2003. An estimated dynamic stochastic general equilibrium model of the euro area. Journal of the European Economics Association 1 (5), 1123-1175.

Smets, F., Wouters, R., 2007. Shocks and frictions in US business cycles: a Bayesian DSGE approach. American Economic Review 97, 586-606. 